Este trabajo ha obtenido el 1.er Premio Estudios Financieros 2016 en la modalidad de Educación y Nuevas Tecnologías. El jurado ha estado compuesto por: don Antonio Bautista García-Vera, don Luis Alberto Guijarro Rojo, doña Ana Hidalgo Tena, doña Blanca Lozano Cutanda y don José Eugenio Martínez Falero. (Los trabajos se presentan con seudónimo y la selección se efectúa garantizando el anonimato de los autores)

\title{
Aplicación de las técnicas despliegue de la función de calidad (QFD) y proceso analítico jerárquico (AHP) a la mejora de la calidad de la formación de posgrado
}

1. Introducción

2. Interés y oportunidad del estudio

3. ¿Es el alumno un cliente en un programa de posgrado?

4. Objetivo

5. Revisión de la literatura sobre calidad educativa y despliegue de la función de calidad y proceso analítico jerárquico

6. Metodología utilizada: despliegue de la función de calidad y proceso analítico jerárquico

7. Estudio realizado

8. Resultados

9. Conclusiones

10. Líneas futuras de investigación

11. Bibliografía

Anexos

\section{Extracto:}

La importancia que en la formación universitaria tienen los principios y políticas de calidad aplicadas a la educación queda patente en el número de organismos locales, nacionales y transnacionales que se dedican, de una u otra forma, a asegurarla, y en el número y volumen de trabajos publicados sobre el tema. Todas las universidades, presenciales y on-line, cuentan con departamentos especializados en el estudio de estas prácticas y políticas, y la literatura académica especializada en esta materia es amplísima. Pero en esta abundante y enorme riqueza de trabajos, estudios, metodologías, técnicas, fantasías y realidades en la que profesores y gestores universitarios, pedagogos, psicólogos, estudiosos del marketing y profesionales de calidad se centran, hay algo, en nuestra opinión básico y elemental, que se olvida frecuentemente: preguntar al alumno, al usuario, al destinatario final (que debería ser el objeto fundamental de nuestro interés) qué aspectos de la educación superior de posgrado tienen verdadero valor para él.

El objetivo del presente trabajo será comprobar la utilidad de las técnicas de análisis multicriterio, en particular el método analítico jerárquico (AHP), unido al despliegue de la función de calidad (QFD), para desarrollar esta tarea en entornos transculturales: los alumnos de posgrados presenciales de la escuela de negocios CEF.

Palabras claves: educación superior, calidad educativa, despliegue de la función de calidad (QFD), proceso analítico jerárquico (AHP). 


\section{Application of quality function deployment (QFD) and analytic hierarchy process (AHP) techniques to improve the quality of postgraduate \\ training}

\section{Abstract:}

The importance of ensuring quality in higher education is evident in the number of local, national and transnational organizations dedicated to this end. The impressive volume of works published on quality in education is further testimony to its importance. All university, traditional or on-line, has a department dedicated to the study of quality, and the academic literature in this area is extremely broad in scope.

Yet in this abundant wealth of studies, methodologies, techniques, fantasies and realities in which professors and university administrators, teachers, psychologists, marketing experts and quality professionals focus their attention, there is in our opinion something basic and elemental which is frequently forgotten: to ask the student, the end user (who should be the fundamental object of our interest) what aspects of postgraduate study he truly values. The goal of the present study will be to verify the usefulness of multi-criteria analysis, and of the analytic hierarchy process (AHP) in particular, attached to quality function deployment (QFD), for performing this task in multicultural environments. The population chosen for our study is comprised of the students enrolled in on-site postgraduate programs at the CEF School of Business.

Keywords: higher education, education quality, quality function deployment (QFD), analytic hierarchy process (AHP).

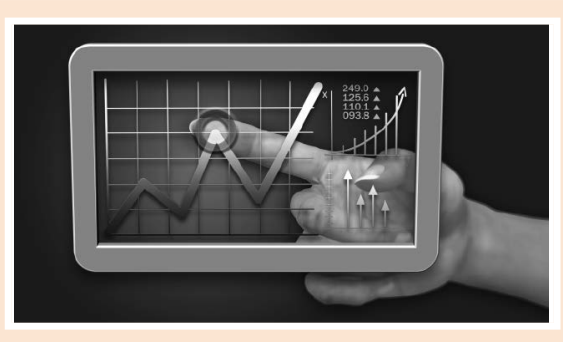

\section{INTRODUCCIÓN}

El objeto de este trabajo será aplicar dos de las metodologías más importantes que se utilizan en la actualidad para el diseño de programas de educación superior totalmente orientados al cliente, dentro del paradigma de la calidad total, el $\mathrm{QFD}^{2}$ y el $\mathrm{AHP}^{3}$, para mejorar el diseño de los programas máster, incorporando de forma rigurosa a este diseño la voz de uno de sus principales clientes: los alumnos que cursan el programa formativo.

En este trabajo solo se plantea el proceso de recoger la voz de este grupo de interés concreto, dejándose para otros trabajos posteriores la aplicación de estas metodologías para recabar de forma fidedigna y operativa la voz de otro de los grandes grupos de interés en estos procesos formativos, la industria a la que se incorporarán los egresados de estos programas.

También podrá observarse cómo el empleo de estas metodologías es razonablemente intuitivo y sencillo para mejorar el diseño de cualquier programa formativo destinado a desarrollar las competencias profesionales de las personas a las que vaya dirigido (programas de formación interna en las empresas, formación profesional, etc.). El requisito básico para que la metodología planteada sea eficaz será la adecuada identificación y selección de los actores en el proceso: los usuarios, los clientes, otros posibles prescriptores y los técnicos especialistas.

\footnotetext{
${ }^{2}$ QFD son las siglas de quality function deployment. En su traducción al castellano (despliegue de la función de calidad) se siguen utilizando habitualmente las siglas de su denominación en inglés, criterio que también se usará en este trabajo.

${ }^{3}$ AHP son las siglas de analytic hierarchy process. En su traducción al castellano (proceso analítico jerárquico) a veces se utilizan, aunque de manera poco frecuente, las siglas PAJ. En este trabajo se usarán las siglas AHP.
} 


\section{INTERÉS Y OPORTUNIDAD DEL ESTUDIO}

Los programas máster en la actualidad están claramente inscritos en el marco universitario, aunque, en España, un gran número de los mismos hayan sido impartidos por instituciones académicas no universitarias, las escuelas de negocios. Pero incluso en estos casos, la gran mayoría de los participantes en los mismos han sido y son titulados universitarios, por lo que siempre han tenido la consideración de estudios de posgrado.

Ya en 1930, José Ortega y Gasset, en su conocido estudio Misión de la universidad, atribuía a la misma las cuatro clásicas funciones que aún todos le reconocen:

- La investigación científica y la preparación de futuros investigadores.

- Desarrollo y transmisión de la "cultura» o sistema de las ideas vivas que cada época posee.

- La enseñanza de las profesiones intelectuales.

- Proporcionar a los estudiantes universitarios la capacidad de mandar.

Abordar el cumplimiento de las dos últimas misiones que Ortega asigna a la universidad en el frenético transcurso del último tercio del siglo pasado, con todos los cambios que se sucedieron de forma constante y en gran medida impredecibles -desde los cambios en la estructura política mundial (crisis del eje este-oeste e irrupción del eje norte-sur), pasando por la radical transformación del entorno socioeconómico (crisis energética, economía globalizada, nacimiento de la sociedad de la información, Chernóbil, etc.), a la aparición de nuevos modelos de organización social (nuevas estructuras familiares, incorporación al trabajo de la generación X, etc.)-, obligó a los profesionales del conocimiento y a los cuadros de mando a adquirir unas habilidades y destrezas donde primara más la rapidez, la oportunidad y la flexibilidad de pensamiento que la absoluta certeza del conocimiento adquirido, es decir, la capacidad para gestionar la incertidumbre.

Sin embargo, la universidad española, con déficits estructurales que dificultan en gran medida su modernización, no estaba en condiciones de dar una adecuada respuesta a estas nuevas necesidades de formación a través de sus estructuras académicas tradicionales (Hernández, Delgado y Pericay, 2013; Núñez, 2013).
Con la intención de atender esta demanda social de contar con unos profesionales y cuadros de mando con mayor flexibilidad de pensamiento y capaces de asumir la responsabilidad de tomar decisiones en situaciones de incertidumbre, es por lo que surgen en España, en la década de los ochenta, las escuelas de negocios, que se convertirán en las grandes protagonistas de la formación de cuadros de mandos y directivos (aún siguen siéndolo) a través de sus programas más característicos, los másteres.

Las escuelas de negocios españolas abordan el reto de la capacitación profesional de los titulados universitarios con una acertada visión de las necesidades sociales del futuro y unos sólidos valores basados en el esfuerzo, la competencia personal de profesores y alumnos, la competitividad entre las instituciones, la flexibilidad de contenidos y metodologías, el rigor, tanto en los conocimientos impartidos, como en los sistemas de evaluación, y el valor necesario para innovar. A esto se suma que las escuelas de negocios poseían en sus inicios una libertad de acción y capacidad de ejecución proporcionada por la no oficialidad de sus programas, lo que las liberaba de las restricciones y limitaciones que encorsetan a la universidad oficial.

Con la intención de atender
esta demanda social de contar
con unos profesionales y
cuadros de mando con mayor
flexibilidad de pensamiento
y capaces de asumir la
responsabilidad de tomar
decisiones en situaciones
de incertidumbre, es por lo
que surgen en España, en la
década de los ochenta, las
escuelas de negocios

Con la intención de atender esta demanda social de contar con unos profesionales $y$ cuadros de mando con mayo flexibilidad de pensamiento y capaces de asumir la responsabilidad de tomar decisiones en situaciones de incertidumbre, es por lo que surgen en España, en la escuelas de negocios 
El resultado obtenido las permite conseguir un grado de reconocimiento y prestigio social muy alto, llegando a figurar muchas de ellas entre las más importantes del mundo (Gasalla, 2010; Hernández, Delgado y Pericay, 2013; Núñez, 2013), siendo este logro consecuencia del esfuerzo que realizan las escuelas de negocios para desarrollar programas formativos totalmente orientados a satisfacer las necesidades que tienen los agentes sociales a los que atienden: perspectiva de carrera profesional sólida para los alumnos y capital intelectual con valor añadido para las empresas.

Este modelo de capacitación profesional para los titulados superiores se mantiene vigente hasta la aprobación de la Ley orgánica 4/2007, de 12 de abril, y del Real Decreto 1393/2007, de 29 de octubre, que establecen la ordenación de las enseñanzas universitarias oficiales y suponen la puesta en marcha en España de los principios del Espacio Europeo de Educación Superior (EEES), legislación por la que se crean en nuestro país los másteres oficiales y, por tanto, están sujetos a la disciplina (y rigidez) universitaria en todos sus aspectos.

A su vez, las nuevas reglas de juego obligan a las escuelas de negocios a vincularse de alguna manera a una universidad oficial para que sus programas puedan incorporarse al sistema universitario español (SUE), el nuevo paradigma de la formación de posgrado.

Las escuelas de negocios españolas abordan el reto de la capacitación profesional de los titulados

universitarios con una acertada visión de las necesidades sociales del futuro y unos sólidos valores basados en el esfuerzo, la competencia personal de profesores y alumnos, la competitividad entre las instituciones, la flexibilidad de contenidos y metodologías, el rigor, tanto en los conocimientos impartidos, como en los sistemas de evaluación, y el valor necesario para innovar

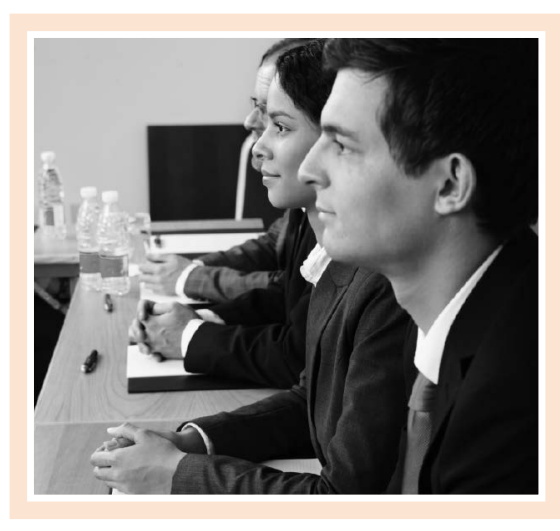

Sin embargo, la necesidad social de desarrollar unos másteres para la formación de mandos con un alto grado de calidad sigue siendo imprescindible, como lo expresa perfectamente el profesor Gasalla (2010, pág. 76), al considerar que «en los tiempos actuales, la formación de directivos desempeña un papel primordial en las organizaciones, ya que si nuestros directivos y colaboradores saben hacer lo que tienen que hacer, es decir, que lo que se les enseña tenga coherencia con lo que se pretende hacer, entonces podremos comenzar a notar la efectividad de haberles enseñado cómo hacerlo».

Este énfasis en transmitir lo necesario para ejercer de forma adecuada la profesión, transformando necesidades profesionales en competencias personales, equivale a tener la capacidad de satisfacer adecuadamente las expectativas que los alumnos traen a las aulas y las competencias que los empleadores necesitan que adquieran. Es decir, aplicar la filosofía de la calidad total a esta formación. Para ello, necesitaremos conocer cuáles son los aspectos fundamentales de un máster que llevan a los estudiantes a elegirlo.

Para alcanzar este objetivo, la combinación de las metodologías QFD y AHP ofrece un marco excelente de actuación, definiendo un modelo que permite mejorar de forma consistente y constante la calidad de los programas de educación superior, imperativo social en esta era de la educación global. 


\section{3. ¿ES EL ALUMNO UN CLIENTE EN UN PROGRAMA DE POS- GRADO?}

Uno de los principales desafíos que tienen los sistemas universitarios para poder aplicar con eficacia la filosofía de la calidad es la dificultad existente para definir con precisión quién es el cliente en los procesos de educación superior (Raharjo et al.,2007; Singh, Grover y Kumar, 2008).

Hay una amplia variedad de grupos de interés en la educación superior: estudiantes, padres y familias, empresarios, personal docente y no docente, Administraciones públicas, organizaciones financiadoras, agencias de acreditación, sociedad en general, etc. Cada uno de estos grupos de interés tiene un punto de vista diferente sobre lo que es una formación de calidad, no existiendo unanimidad respecto al papel que debe jugar cada uno de estos colectivos. Esta situación conduce a que nos preguntemos quién o quiénes son los clientes en un máster. Y la respuesta que obtengamos determinará quiénes debieran ser los interlocutores esenciales en el diseño de los mismos.

En general, existen pocas dudas respecto al papel clientelar que juegan en la formación de posgrado tanto los profesores, como las instituciones educativas y los empleadores.

El problema se suele presentar a la hora de considerar al estudiante un cliente de pleno derecho y, por tanto, si se debe tener en cuenta la opinión del mismo para el diseño y posterior evaluación de estos programas. ¿Están los estudiantes cualificados sobre el diseño de un máster? ¿Sus opiniones son fiables o están normalmente sesgadas por la «tendencia al mínimo esfuerzo»? ¿Deben evaluar a sus profesores con el inevitable riesgo de decidir sus valoraciones en función de los resultados académicos conseguidos?

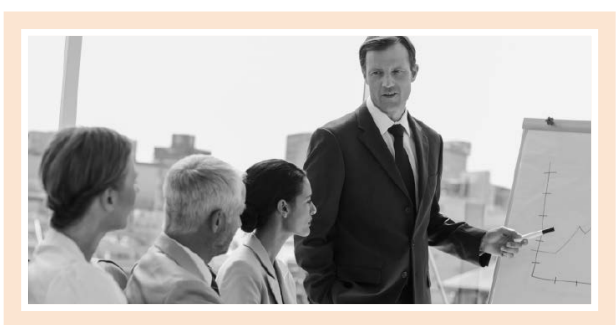
Uno de los principales desafíos que
tienen los sistemas universitarios para poder aplicar con eficacia la filosofía de la calidad es la dificultad existente para definir con precisión quién es el cliente en los procesos de educación superior

En muchos casos, profesores e instituciones educativas consideran que los alumnos carecen de los conocimientos necesarios para influir en el contenido de los cursos y que la «satisfacción de sus necesidades» no conduce necesariamente a la calidad del proceso educativo. Esta creencia se basa en la suposición de que los estudiantes solo se preocupan de su comodidad en el proceso de estudio, en lugar de focalizarse en su aprendizaje y desarrollo. Con frecuencia, el profesorado y los responsables de las instituciones educativas piensan que ellos saben mejor que los propios estudiantes lo que estos necesitan, mientras que los estudiantes no pueden saber, al menos en las primeras etapas de su proceso educativo, lo que es más conveniente para su formación (Singh, Grover y Kumar, 2008).

Esta dificultad para considerar al estudiante como cliente del proceso educativo la comparten los servicios sanitarios a la hora de considerar como cliente al enfermo:

«(...) aun cuando aceptemos inicialmente que el cliente del hospital es el paciente, no tardaremos en descubrir que es un tipo de relación proveedor-cliente especial (...) Y la característica más singular es que, una vez admitido en el hospital, el paciente se convierte en un componente (materia prima) esencial para la producción del producto hospitalario: pacientes con mejor salud» (Valor y Ribera, 1988, pág. 177). 
Esta propiedad de irse transformando sucesivamente de «cliente» en «materia prima», de «materia prima» en «producto» y de «producto», nuevamente, en "cliente», ya que el proceso contratado transforma en sí mismo al cliente, es compartida tanto por los estudiantes dentro de un proceso educativo, como por los pacientes dentro de un proceso asistencial y hace que sea especialmente compleja la consideración de ambos sujetos como clientes. La figura 1 representa esta transformación de los roles por los que pasa el estudiante.

Probablemente, una medida cautelar imprescindible para que en esta transformación final el "procesando» vuelva a ser el «rey de oros» (o al menos, el de copas), y evitar que se convierta en "rey de bastos», sea conocer en profundidad qué expectativas trae al proceso en cuestión.

En todo caso, existe una importante tendencia en la literatura especializada a considerar que, si bien el estudiante a veces puede no ser la persona más cualificada para opinar sobre el contenido de un plan de estudios, sí tendrá mucho que decir sobre el profesorado que espera encontrar, su comportamiento, sus habilidades de comunicación, su accesibilidad, su experiencia profesional, los recursos utilizados, la organización del horario, etc. (Singh, Grover y Kumar, 2008) ${ }^{4}$.

En refuerzo de esta idea, Marsh y Roche (1997) encontraron que las evaluaciones que realizaban los estudiantes sobre la enseñanza recibida eran fiables, estables, fundamentalmente dirigidas a los profesores, más que al curso realizado, y relativamente poco afectadas por factores tales como la indulgencia en la calificación, la carga de trabajo, el tamaño de la clase, temas de interés previos, etc., y son sumamente útiles para mejorar la eficacia de la enseñanza cuando se incluyen las preguntas adecuadas.

En nuestro caso, además, si se observa la descripción de la muestra de estudiantes consultados (edad, experiencia profesional, situación profesional, etc.), difícilmente podría argumentarse que la mayoría de ellos no poseen la competencia necesaria para opinar, incluso sobre la bondad del programa cursado.

\footnotetext{
${ }^{4}$ Lo que no impide que, en algún caso, se pueda llevar este planteamiento a extremos algo extravagantes. Por ejemplo, Sa y Saraiva (2001) trataron de incluir a niños de guardería como clientes (citado por Raharjo et al., 2007).
}

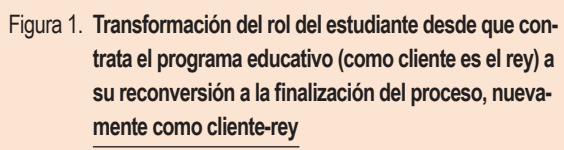

Figura 1. Transformación del rol del estudiante desde que contrata el programa educativo (como cliente es el rey) a su reconversión a la finalización del proceso, nuevamente como cliente-rey

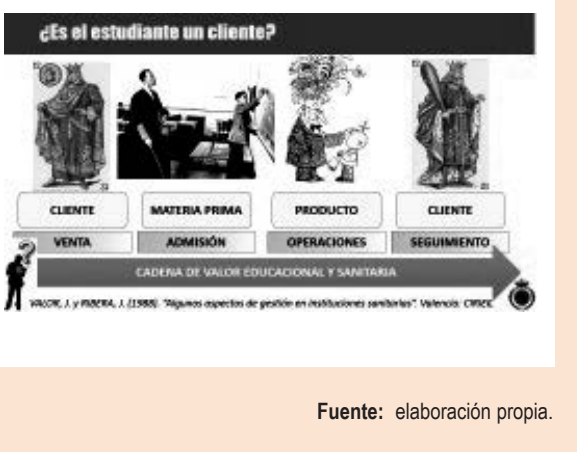

Por lo tanto, en este trabajo vamos a escuchar la voz de uno de los principales clientes de un máster, el estudiante (Raharjo et al., 2007; Singh, Grover y Kumar, 2008).

\section{OBJETIVO}

Aplicar dos de las metodologías más potentes de las políticas de calidad total (QFD y AHP) para conocer, cuantificar y priorizar las expectativas de los alumnos de programas máster de dirección y gestión empresarial (modalidad presencial) de una importante universidad y escuela de negocios al inicio del programa, es decir, sus expectativas iniciales. El objetivo final será obtener un «Mapa de Calidad Esperada» por los estudiantes de másteres presenciales de la escuela de negocios CEF.

El objetivo final será obtener un
«Mapa de Calidad Esperada»
por los estudiantes de másteres
presenciales de la escuela de
negocios CEF




\section{REVISIÓN DE LA LITERATURA SOBRE CALIDAD EDUCATI- VA Y DESPLIEGUE DE LA FUNCIÓN DE CALIDAD Y PROCESO ANALÍTICO JERÁRQUICO}

La metodología QFD es una manera muy potente de escuchar mejor la voz del cliente (VoC), tanto en las industrias de fabricación, como en las empresas de servicios, siendo una de las metodologías más utilizadas en la implantación de la cultura de la calidad total (TQM), principalmente en procesos industriales; si bien, en las dos últimas décadas, hemos podido comprobar cómo está teniendo una amplia utilización en el mundo universitario para incorporar al mismo la cultura de la orientación al cliente (Raharjo et al., 2007).

En el ámbito de la educación superior esta metodología se ha utilizado, sobre todo, para incorporar la voz de los empleadores y profesionales de la industria, junto a la de los profesores y alumnos. Así, se revisaron 42 trabajos en los que se aplicaron de forma sistemática las metodologías QFD y AHP (bien de forma independiente, bien en combinación) a problemas del entorno universitario, comprobándose cómo la aplicación de estas técnicas a la educación superior trataba de mejorar los diseños formativos en todo tipo de aspectos:

- Diseño de programas formativos teniendo en cuenta a todos los agentes implicados: tejido industrial, profesores y estudiantes (21 estudios).

- Medir la satisfacción de los clientes de los programas formativos (11 estudios).

- Identificar y evaluar la potencia formativa de diferentes técnicas de enseñanza (2 estudios).

- Analizar qué técnicas de calidad se aplican a la educación superior (4 estudios).

- Selección de los materiales de estudio de programas formativos (1 estudio).

- Aplicación de un modelo WEB-QFD para mejorar el desarrollo de un programa formativo (1 estudio).

- Análisis de los factores que estaban provocando pérdidas de estudiantes en una universidad (1 estudio).

- Asignación de recursos económicos a los programas formativos (1 estudio).

La relación de estos trabajos puede consultarse en el anexo 2.

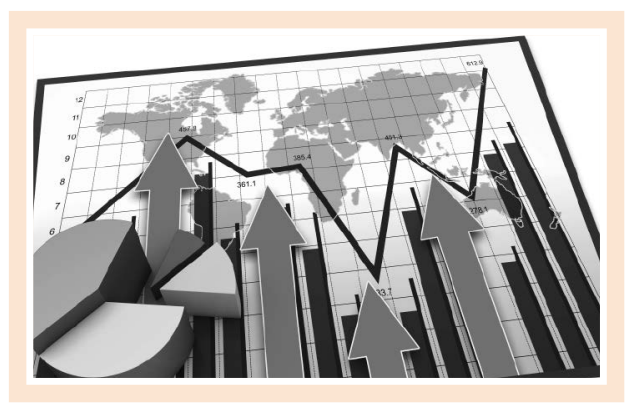

A la vista de estos trabajos, aparecen algunas reflexiones que podrian ser temas de interés para futuras investigaciones. En primer lugar, podemos observar que las universidades de todo el mundo están empezando a aplicar las técnicas de mejora industrial y empresarial al diseño y gestión de los programas universitarios para adaptarlos a las necesidades sociales (empresas y estudiantes). Y si observamos la figura 2, podemos ver que los lugares de realización de los 43 trabajos revisados son:

- Europa: ocho (2, en 1998; 1, en 1999; 1, en 2006; 1 , en 2009;1, en 2010; y 2, en 2011).

- EE. UU.: ocho (1, en 1994; 1, en 1995; 2 en 2001; 2 , en 2002; 1, en 2006; y 1, en 2011).

- Países islámicos: ocho (2, en Turquía, en 1998 y 2005; 1, en Paquistán, en 2012; 3 , en Irán, en 1998, 2012 y 2013; 1, en Arabia Saudita, en 2003; y 1, en Dubái, en 2011).

- Extremo Oriente: puede llamar la atención que 19 publicaciones pertenezcan a países de esta zona del mundo [3, a Hong Kong, en 1998, 2002 y 2009; 2 , a Singapur, en 2007 y 2011; 5 , a la India, en 2003, 2004, 2006 (con dos publicaciones) y 2008; 6 , a China (3, en 2010; y 3, en 2011); 2, a Taiwán, en 2004 y 2012; y 1, a Bangladesh, en 2012].

Quizás deberíamos prestar más atención al ingente esfuerzo que están realizando las economías emergentes por dotarse de una universidad de calidad si vamos a tener que competir con ellas en un mundo global. 
Figura 2. Número de trabajos evaluados en las dos últimas décadas respecto a la aplicación de las metodologías QFD y AHP aplicadas a la educación superior (por lugar de procedencia y año)

\begin{tabular}{|c|c|c|c|c|}
\hline Año & USA & EUROPA & P. ISLÁMICOS & EXTREMO ORIENTE \\
\hline 1994 & & $\begin{array}{l} \\
\end{array}$ & $\dot{\vdots}$ & 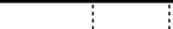 \\
\hline 1995 & & & $\dot{i}$ & \\
\hline 1998 & & & & 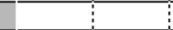 \\
\hline 1999 & & & & 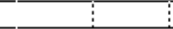 \\
\hline 2001 & & & & 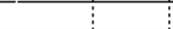 \\
\hline 2002 & & & & 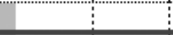 \\
\hline 2003 & & & & 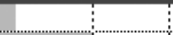 \\
\hline 2004 & & & & 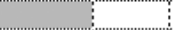 \\
\hline 2005 & & & & 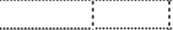 \\
\hline 2006 & & & & \\
\hline 2007 & & & & \\
\hline 2008 & & & & \\
\hline 2009 & & & & \\
\hline 2010 & & & & \\
\hline 2011 & & & & \\
\hline 2012 & & & & \\
\hline 2013 & & ¿ & & 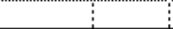 \\
\hline
\end{tabular}

\section{METODOLOGÍA UTILIZADA: DESPLIEGUE DE LA FUNCIÓN DE CALIDAD Y PROCESO ANALÍTICO JERÁRQUICO}

\subsection{Despliegue de la función de calidad y escuchar la «voz del cliente»}

El QFD puede definirse como «un sistema estructurado que permite identificar necesidades y expectativas de los clientes (VoC) y traducirlas al lenguaje de la organización, esto es, a requerimientos de calidad internos» (Guzmán, 2013, pág. 1), ayudando de esta manera a determinar los requisitos críticos que se tienen que desarrollar para lograr la satisfacción total del cliente. Así, la eficacia que se alcance en la aplicación del QFD dependerá del grado de la satisfacción de todas las partes interesadas.

La metodología QFD la desarrollaron los doctores Shigeru Mizuno y Yoji Akao, y está estrechamente ligada a la filosofía de la calidad total del doctor Kaoru Ishikawa, que pretende fundamentalmente:
- Conocer las necesidades del cliente, tanto explícitas, como implícitas, escuchándolas de primera mano.

- Traducir estas necesidades formuladas en los diseños de los productos y servicios que el cliente recibe.

- Integrar todas las funciones de la organización en esta dirección para conseguir la satisfacción de los objetivos del cliente (QFD Institute, 2013).

Esta metodología es lo que se conoce como escuchar "la $\mathrm{VoC}$ » y permite, como ya se ha dicho, integrar tanto los requisitos técnicos de un determinado diseño (la voz de los expertos), como las expectativas del cliente.

Con frecuencia, la metodología QFD se representa en un diagrama conocido como la matriz básica de la QFD, o también la casa de la calidad (HOQ) (Johnson, 2003; Raharjo et al., 2007). 
En este diagrama (véase figura 3):

- El eje X recoge las especificaciones y necesidades (explícitas e implícitas) del cliente.

- El eje Y detalla los requisitos técnicos que deben dar respuesta a esas necesidades.

En la intersección de ambos ejes se encuentra el diseño buscado.

Siguiendo a Guzmán (2013), un proceso que trate de escuchar la VoC (QFD) debe incluir las siguientes fases:

- Identificar y jerarquizar a los clientes. No todos los clientes tienen necesariamente la misma importancia en cada proceso.

- Conocer sus expectativas. Para ello, puede ser utilizado cualquiera de los métodos disponibles para captar la información necesaria: grupos de discusión, análisis de quejas y reclamaciones, encuestas, publicaciones, etc.

- Convertir la información obtenida de los clientes en descripciones verbales específicas.

- Elaborar y distribuir un cuestionario a los clientes para que evalúen la importancia que tienen para ellos, los factores estudiados.

- Elaborar el mapa de calidad esperada (despliegue de la calidad-demanda): prioridades que los clientes atribuyen a cada uno de los factores previamente definidos. Para desarrollar esta fase, la metodología más utilizada en los procesos de QFD es el proceso analítico jerárquico (AHP), que también utilizaremos nosotros.

- Realizar el despliegue de las características de la calidad: planificación de las mejoras de los productos o servicios analizados, en función de las expectativas de los clientes. Este último punto quedará fuera del alcance del presente trabajo.

Para Jimeno (2013), el QFD comienza elaborando la «lista de los QUÉ», las expectativas y necesidades de los clientes, para, en la siguiente fase, realizar el «análisis de los QUÉ», clasificándolos por la importancia que estos aspectos tienen para los clientes.

Como se ha podido comprobar, la filosofía que subyace al QFD es muy sencilla y fácilmente comprensible. Las dificultades se presentan en los aspectos operativos de su implantación, ya que desarrollar cada uno de los sencillos pasos anteriores genera dificultades técnicas que, en algún caso, pueden tener bastante complejidad matemática. El proceso de implantación operativa del QFD se suele acometer con el auxilio de la metodología AHP.
Figura 3. Matriz básica del QFD

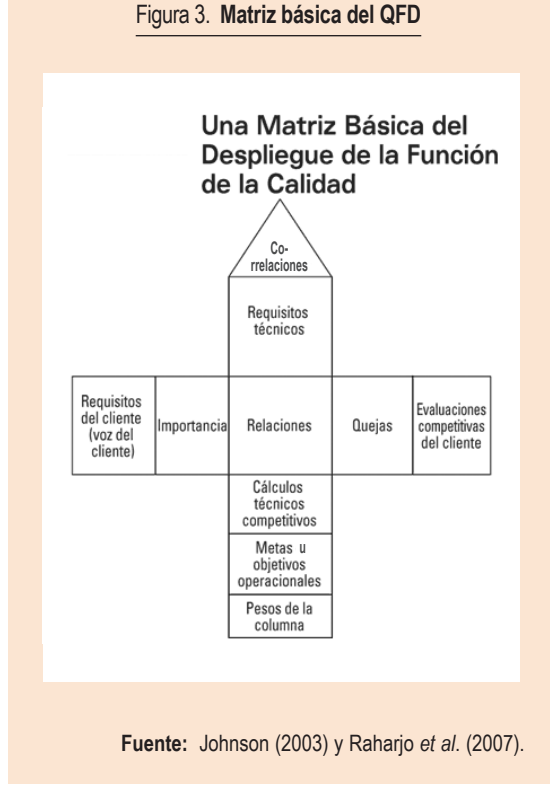

\subsection{Analizar lo que dice el cliente: pro- ceso analítico jerárquico}

La metodología AHP es un método de decisión multicriterio discreto (DMD), es decir, es un método de ayuda a la toma de decisiones, elegir 0 priorizar entre un conjunto finito de alternativas, cuando estas poseen muchos factores o atributos relevantes que incluso pueden ser contradictorios entre sí. En estos casos, no hay una solución «ideal en el sentido de que sea la alternativa mejor valorada en todas sus dimensiones 0 atributos. La solución pasa por encontrar el «óptimo paretiano» o alternativa que en conjunto sale mejor evaluada, aunque puede no salir bien considerada en alguno de los factores que la caracterizan.

El AHP está considerado como el método de decisión multicriterio que permite una discriminación mejor del proceso de decisión, gracias a la posibilidad que tiene de incorporar tantos niveles de criterios y subcriterios como sea necesario (Zanazzi, 2003). 
El AHP lo desarrolló Saaty en 1980 y trata de obtener un método de análisis que reproduzca la capacidad innata que tenemos las personas de emitir juicios bien fundados sobre pequeños problemas de la forma más fidedigna posible.

En esencia, el método recoge las prioridades o preferencias que tiene una persona sobre un conjunto de ítems o elementos para, posteriormente, poder seleccionar el más conveniente, poder clasificar estos ítems en función de algún criterio, o bien ordenarlos en función de las preferencias encontradas (Moreno, 2002; Saaty, 1980 y 1998; Zanazzi, 2003).

Saaty inicia su método con la definición del problema y su estructuración (la parte más creativa de una decisión), formulando una jerarquía de subproblemas. A continuación, el AHP prioriza las subalternativas generadas en función de los criterios de preferencia definidos previamente, para terminar integrando todas las prioridades facilitadas a los subproblemas analizados en una solución global (Zanazzi, 2003).

Saaty considera que el AHP se construye o asienta sobre siete principios básicos, los «siete pilares del AHP» (Saaty y Vargas, 2001):

1. En el AHP, cada decisor compara cada una de las alternativas con todas las demás, dos a dos, expresando de esta forma su preferencia sobre ellas.

2. Para realizar las comparaciones entre alternativas, el AHP utiliza una escala de razón (proporciones).
Por ejemplo, la escala de un mapa es una escala de razón.

3. El AHP utiliza un complejo modelo matemático para obtener las prioridades de los factores, basado en el álgebra de vectores.

4. Las alternativas se valoran utilizando la escala fundamental de Saaty (que es una escala de razón). Al utilizar siempre números positivos para las comparaciones se eliminan las ambigüedades que las personas tenemos cuando comparamos elementos negativos, muy próximos al cero o excesivamente grandes $(\infty)$.

5. Para obtener la síntesis de las prioridades locales para el cálculo de la prioridad global, el AHP utiliza un proceso de suma ponderada. Esto permite transformar un problema multidimensional o multicriterio en una escala de razón normalizada unidimensional y que representa las prioridades globales del problema analizado. Es decir, permite comparar prioridades sobre dimensiones diferentes.

6. El AHP asegura que se conservan las prioridades ya obtenidas entre las alternativas comparadas si se introducen o suprimen nuevas alternativas.

7. EI AHP permite construir decisiones grupales a partir de las decisiones individuales. Esta decisión grupal es compatible con las decisiones individuales y el proceso está bien justificado matemáticamente, por lo que facilita la obtención de consensos.

Tabla 1. Escala fundamental de Saaty

\begin{tabular}{|c|l|}
$\begin{array}{c}\text { Valor o intensidad } \\
\text { de la comparación }\end{array}$ & Se puntúa asi una comparación entre dos factores cuando: \\
\hline 1 & Ambos tienen la misma importancia para alcanzar el objetivo \\
3 & Cuando uno de los factores tiene claramente más importancia que el otro \\
5 & Cuando uno de ellos tiene mucha más importancia que el otro factor \\
7 & Cuando uno de los factores tiene gran importancia respecto al otro \\
9 & La importancia de uno de los factores sobre el otro es extrema \\
\hline
\end{tabular}




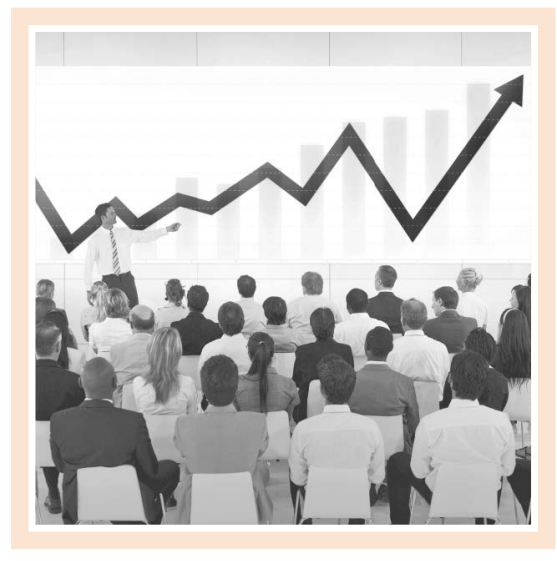

\subsubsection{Metodología de aplicación del proceso analítico jerárquico}

La estructura clásica del AHP formulada por Saaty ha sido ampliada y mejorada en los últimos años incorporando a la metodología procesos de análisis de sensibilidad y robustez de los datos y una fase final de toma de decisiones específica, basada en el aprendizaje y la negociación entre actores. Reformulando estos planteamientos, la metodología que seguiremos en la aplicación del método AHP puede esquematizarse de la siguiente manera (Moreno, 2002; Saaty, 1980 y 1998; Zanazzi, 2003; Viswanadhan, 2005):

\section{A) Formulación y descripción del problema}

\section{a) Identificar el objetivo general (nivel 1)}

En este apartado se trata de contestar a las siguientes preguntas: ¿qué es lo que se quiere lograr con el planteamiento del problema?; ¿cuál es la pregunta principal que hay que contestar? Si es relevante, en este momento se identifican los plazos de tiempo que afectan a la decisión.

El objetivo general se coloca en el primer nivel de la pirámide de criterios (nivel 1). En nuestro caso, este nivel $1 \mathrm{u}$ objetivo general se recoge en la pregunta «cuál es la calidad esperada por los estudiantes de un programa máster presencial». b) Identificar a los actores involucrados en la decisión

En general, habrá cuatro tipos de actores (o expertos) en la resolución de este tipo de problemas:

- Propietarios del problema o gerentes. Aquellas personas que tienen la responsabilidad de solucionar o mejorar el problema. En nuestro caso, la dirección de la escuela de negocios y universidad.

- Facilitadores o técnicos. Expertos en la metodología y su aplicación. Son técnicos que garantizan el correcto desarrollo del proceso, la metodología. En nuestro caso, el equipo que realizó el estudio.

- Evaluadores o prescriptores. Expertos en el problema en general. Definen los «aspectos relevantes» (estructura jerárquica del problema, criterios, alternativas y subcriterios, etc.). Normalmente serán prescriptores en el entorno. En nuestro caso, se utilizaron tres fuentes prescriptoras: literatura especializada, opinión de los jefes de estudio y grupos de discusión con egresados.

- Decisores o clientes. Aquellas personas de las que se trata de obtener la opinión. Son los encargados de realizar la evaluación y priorización de los atributos analizados. En nuestro caso, los estudiantes de los másteres encuestados.

\section{B) Modelización. Definición de la estructura jerárquica}

El objetivo de esta fase es identificar, primero, los criterios, atributos o dimensiones que definen o intervienen en el objetivo general y, posteriormente, las alternativas y subcriterios relevantes que constituyen la esencia de estos criterios y cumplen con los requisitos, restricciones y limitaciones conocidas o previsibles (nivel 3 y siguientes, si los hay). En general, la descomposición en niveles se realiza desde los factores más generales a los más específicos y concretos. En el último nivel se especifican los elementos de decisión entre los que se debe seleccionar.

La jerarquía obtenida debe ser completa, representativa (incluye todos los atributos relevantes), no redundante y minimal (no incluye los aspectos irrelevantes), y para elaborarla, normalmente, se precisará llegar a acuerdos entre los expertos que la definen y de una buena dosis de creatividad (Moreno, 2002; Saaty, 1980 y 1998).

En nuestro caso, esta estructura jerárquica la compusieron las dimensiones principales que se consideraron relevantes para analizar la calidad de un máster (6) y los factores que se consideraron relevantes dentro de cada dimensión ( 6 por dimensión). 


\section{C) Valoración}

El objetivo final de esta fase es conocer el grado de prioridad o importancia que para cada uno de los «decisores o clientes» tienen los criterios que componen el objetivo general formulado, según una escala de razón. Es decir, saber qué criterios son más importantes y cuánto más lo son que los demás.

Para resolver este problema, el AHP desarrolla el proceso en cinco fases:

1. Efectuar las comparaciones entre pares de los elementos de decisión por las personas responsables de formular las priorizaciones, valoraciones o juicios.

2. Elaborar la matriz de comparación de juicios de cada decisor.

3. Cálculo de las «prioridades locales» de las alternativas (factores del último nivel) para cada experto (decisor o cliente).

4. Determinar si los datos de entrada satisfacen una prueba de consistencia. Si no es así, volver al paso 1 y rehacer las comparaciones entre pares de elementos.

5. Determinación de las «prioridades globales (grado de influencia de cada una de las alternativas del último nivel, sobre el objetivo general) y totales (influencia de los criterios principales, nivel 2, sobre el objetivo general)».

En nuestro caso, este resultado constituyó el «mapa de calidad esperada» de cada uno de los estudiantes encuestados.

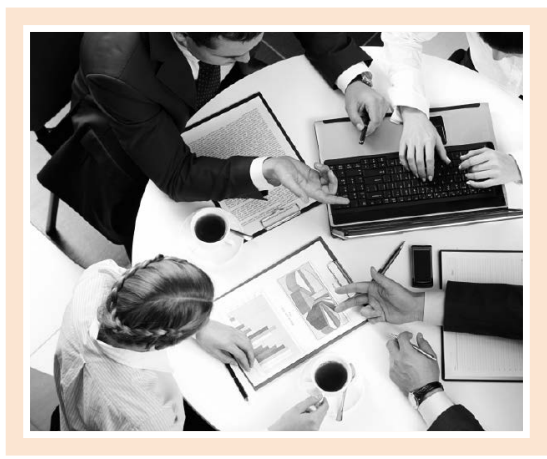

D) Agregación de los juicios efectuados por los diferentes decisores o clientes

En el caso de que en la fase de elaboración de la matriz de comparación de juicios contemos con la opinión de varios expertos, se recomienda la utilización de la media geométrica de las opiniones de todos ellos, frente a la media aritmética. La metodología también permite tener en cuenta que las opiniones de los decisores o clientes puedan tener un peso específico diferencial, lo que no fue nuestro caso (Moreno, 2002; Raharjo et al., 2007; Saaty, 1980 y 1998; Viswanadhan, 2005; Zanazzi, 2003).

En nuestro caso, el resultado de esta fase lo constituyó el «mapa de calidad esperada» de cada uno de los grupos de interés y el de toda la muestra de estudiantes. Nuestro objetivo final.

\section{E) Análisis de sensibilidad, robustez e incertidumbre}

Esta fase del AHP trata de reducir la incertidumbre existente en el proceso de planificación y evaluación seguido, atendiendo a tres niveles: efectividad o validez, eficacia o robustez y eficiencia, estabilidad o sensibilidad. Como en la literatura sobre decisión multicriterio y, en concreto, sobre AHP, existe una relativa falta de consenso del significado o interpretación de cada uno de ellos y sobre el procedimiento más adecuado para realizarlos, nosotros no lo calcularemos en nuestro estudio (Moreno et al., 1998).

\subsubsection{Ventajas del proceso analítico jerárquico}

Como ya hemos tenido ocasión de ver hasta el momento, las ventajas que presenta el AHP como método de decisión multicriterio sobre otras metodologías han sido ampliamente resaltadas (Moreno, 2002; Saaty, 1980 y 1998; Viswanadhan, 2005; Zanazzi, 2003). Entre estas ventajas podemos destacar su forma de abordar el problema descomponiéndolo en partes más manejables, lo que emula (según Saaty) la forma que tienen las personas de abordar este tipo de problemas complejos. Esto hace que su utilización sea muy intuitiva y comprensible, incluso para participantes profanos. Además, la posibilidad de integrar en el proceso de evaluación dimensiones tangibles (medidas absolutas) e intangibles (atributos psicológicos y emocionales) aumenta la riqueza y transparencia de las mismas. 
Otro tipo de ventajas se desprenden de su estructura matemática, muy fundamentada en la teoría de grafos y el cálculo matricial, lo que permite obtener con objetividad resultados numéricos de las preferencias encontradas, lo que facilita el consenso entre grupos de trabajo y la posible revisión de las mismas a lo largo del tiempo. Como contrapartida, requiere tener unos conocimientos mínimos de álgebra vectorial para ejercer los roles técnicos, competencia que no es necesaria para los restantes actores del proceso.

El grado de compromiso y colaboración que esta metodología despierta en los participantes es muy alto. Al participante se le exige un grado de concentración y atención durante la cumplimentación del cuestionario mayor del que es habitual tener en este tipo de tareas. Hacer comparaciones ponderadas dos a dos exigía focalizarse mucho en la tarea y admitía pocas distracciones. Esto hacia que los participantes, posteriormente, tuvieran mucha curiosidad y mostraran mucho interés por conocer los resultados de la investigación. Esto lo convierte en un método altamente atractivo en procesos de evaluación y diseño que requieran compromiso y aceptación por parte de los interlocutores que participan en el proceso.

\subsubsection{Problemas del proceso analítico jerárquico}

No obstante, el método del AHP no ha dejado de recibir críticas en muchos de sus planteamientos. Zanazzi (2003) realiza una recopilación de las principales dificultades o problemas que puede presentar el método AHP. Entre ellas destacan posibles dificultades asociadas a la estructura jerárquica del proceso de decisión; dificultades por el sistema utilizado para la valoración de las preferencias (escala fundamental de Saaty); también se han formulado críticas a la garantía que ofrece la metodología con la reversión de rangos (posibles inconsistencias); el método ha recibido críticas por el sistema de normalización de las valoraciones utilizado y por el sistema de agregación de los juicios empleado.

En todo caso, no es objeto del presente trabajo realizar un análisis de las mismas, dejando simplemente constancia de su existencia.

\section{EI QFD puede definirse como «un sistema estructurado que permite identificar necesidades y expectativas de los clientes (VoC) y traducirlas al lenguaje de la organización, esto es, a requerimientos de calidad internos»}

\subsubsection{Aplicaciones del proceso analítico jerárquico}

La metodología AHP se aplica a una amplia gama de problemas y es la herramienta más característica en la implantación de programas de mejora basados en el QFD [QFD Institute, 2013; Asociación Latinoamericana de QFD (QFDLAT), 2014]. Entre sus aplicaciones cabe destacar su utilización para la mejora de procesos de gestión de recursos humanos (por ejemplo, diseño de políticas salariales, evaluación del desempeño en recursos humanos, determinación de las capacidades clave de una empresa, implementación de metodologías de calidad total, diseño y evaluación de programas de formación, etc.) y su aplicación a problemas logísticos.

Respecto a la aplicación en educación superior de la metodología AHP, dentro del marco de programas QFD, ya vimos cómo la combinación de las metodologías QFD y AHP ha tenido amplio eco en el diseño de la educación superior y la especial intensidad de empleo con la que la están aplicando las economías emergentes, a pesar de la complejidad de la misma y sus altos costes de aplicación en muestras muy amplias de personas.

Esta amplitud de utilización va a tener su explicación en las ventajas que posee esta herramienta a la hora de aplicarla en ámbitos académicos: estructuración «psicológica» del problema; capacidad para integrar aspectos tangibles e intangibles del mismo; posibilidad de obtener «decisiones grupales»; facilidad de interpretación de los resultados obtenidos, al proporcionar valoraciones numéricas de las prioridades, necesitar un conocimiento matemático generalmente asumible, etc. Esto va a proporcionar a los gestores de los programas universitarios la información necesaria para responder mejor a las necesidades futuras tanto de los estudiantes como del tejido empresarial y social, lo que permite a los centros de educación superior estar alerta, activos, y tener visión de futuro (Kuo, 2006). 


\section{ESTUDIO REALIZADO}

\subsection{Identificar y seleccionar a los «actores» en el proceso}

Ajustándose a la metodología AHP, los actores en nuestra investigación serán:

\section{- Propietarios del problema o} gerentes. Este rol está desempeñado por la estructura directiva de la escuela de negocios y universidad que ha identificado $y$ formulado el problema y, en última instancia, decidirá qué decisiones tomar a la vista de los resultados.

- Facilitadores o técnicos. Esta función la desempeña el equipo técnico que ha desarrollado este trabajo.

\section{- Evaluadores o prescriptores.}

Su misión principal es identificar y seleccionar los ítems que posteriormente deberán evaluar los estudiantes (decisores). En nuestro estudio utilizamos tres fuentes prescriptoras en el proceso:

- Revisión de la literatura científica.

- Grupos de discusión.

- Directores de los másteres a los que pertenecen los estudiantes consultados.

Su papel se analizará con mayor detenimiento en el apartado identificación de ítems críticos.

- Estudiantes o decisores. Muestra de estudiantes a los que se les consulta sobre sus prioridades respecto a los criterios evaluados.

\section{Figura 4. Descripción de la muestra utilizada}

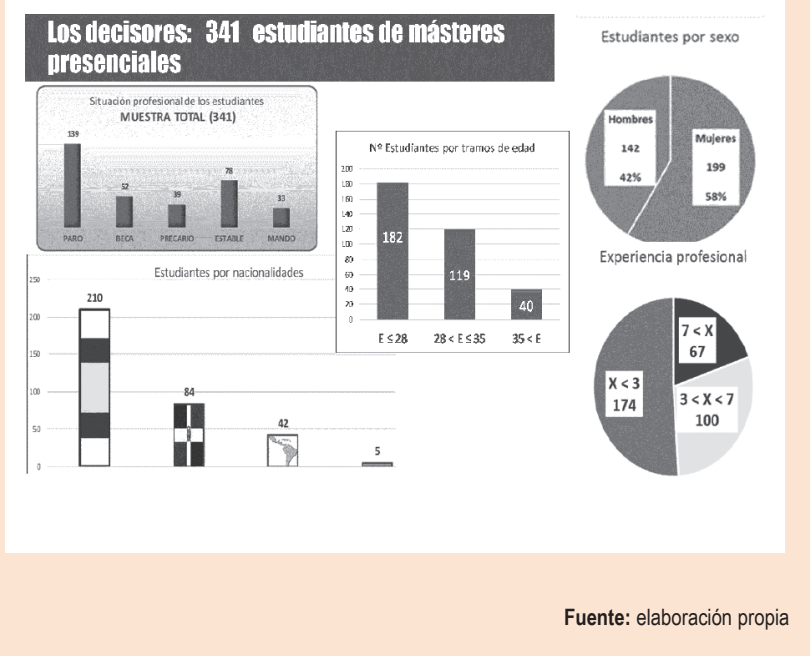

\subsection{Descripción de la muestra de estudiantes consul- tados}

La muestra consultada estaba compuesta por $341^{5}$ estudiantes de programas máster en su modalidad presencial. Todos habían comenzado el máster en octubre de 2013 y se les consultó sobre sus prioridades al inicio del programa.

Las variables demográficas que se tomaron en cuenta fueron aquellas que en la definición del problema se consideraron que podían generar segmentos diferenciados de clientes $y$, por tanto, necesidades y expectativas diferentes respecto al programa que hay que desarrollar. Las variables analizadas y la distribución de los estudiantes en las mismas fueron (véase figura 4):

- Edad:

- Menores de 28 años $182(54 \%)$

- Entre 28 y 35 años $119(35 \%)$

- Mayores de 35 años $40(11 \%)$

\footnotetext{
${ }^{5}$ La muestra inicial se compuso de 363 estudiantes, pero se tuvieron que descartar 22 cuestionarios por defectos de forma.
} 
- Sexo:

- Mujeres

$199(58 \%)$

- Hombres

- Nacionalidad:

- Españoles

- Dominicanos ${ }^{6}$

- Latinoamericanos (no dominicanos) .....

$42(12 \%)$

- Del resto del mundo

- Grado de experiencia profesional del estudiante:

- Menos de tres años de experiencia........

$174(51 \%)$

- De tres a siete años de experiencia ...... 100 (29\%)

- Más de siete años de experiencia ......... $67(20 \%)$

- Situación profesional en el momento de realizar el cuestionario (comienzo del máster) del estudiante:

- En paro

- Realizando prácticas en empresas7......

$52(15 \%)$

- Trabajo precario o claramente por debajo de la capacitación y experiencia del estudiante

- Trabajo razonablemente estable y/o razonablemente acorde a la capacitación y experiencia de los estudiantes, aunque sin responsabilidad sobre otras personas

$78(23 \%)$

- Mando o directivo

\footnotetext{
${ }^{6}$ Estos estudiantes son becados por el Gobierno de la República Dominicana y constituyen un grupo estratégico para la escuela de negocios. Por eso, además de su importante número, se estudiaron sus necesidades de forma diferencial.

7 Se les pedía expresamente en qué situación administrativa estaban dentro de la empresa en que estuvieran trabajando. Muchos estudiantes, especialmente los extranjeros, si no tienen permiso de trabajo, no pueden desarrollar una actividad profesional si no es bajo la situación administrativa de «Convenio de Colaboración Formativa» o beca. Es conocido por todos cómo estudiantes que tienen ya un importante grado de experiencia profesional tienen que seguir realizando «prácticas» si quieren tener acceso a la empresa española.
}

\subsection{Identificación y selección de ítems que se van a evaluar}

En la fase de identificación y selección de ítems, se utilizaron cuatro procesos complementarios y sucesivos:

- Revisión de la literatura científica.

- Directores de los másteres.

- Realización de grupos de discusión con una muestra cualificada de estudiantes.

- Depuración del cuestionario y elaboración del listado definitivo de ítems.

\subsubsection{Análisis de la literatura científica}

El objetivo de este proceso fue determinar un primer grupo amplio de los factores clave para considerar un proceso de posgrado de calidad sobre el que hubiera consenso en la literatura científica.

En esta fase de la investigación se manejaron cuatro investigaciones de cortes similares a la nuestra:

- Owlia y Aspinwall (1998).

- Singh, Grover y Kumar (2008).

- Tsinidou, Gerogiannis y Fitsilis (2010).

- Agencia de Calidad, Acreditación y Prospectiva (ACAP) (2006).

Del análisis comparativo de estas cuatro fuentes se determinó un primer listado de variables compuesto por siete factores principales, con ocho subfactores en cada uno de ellos.

\subsubsection{Análisis por los directores de más- teres}

El objetivo de esta fase era adecuar la clasificación obtenida en el punto anterior a la realidad de la escuela de negocios, completándola con los ítems necesarios.

Como resultado de esta fase, el cuestionario se simplificó a seis factores principales, cada uno de ellos con siete subfactores de segundo orden. 


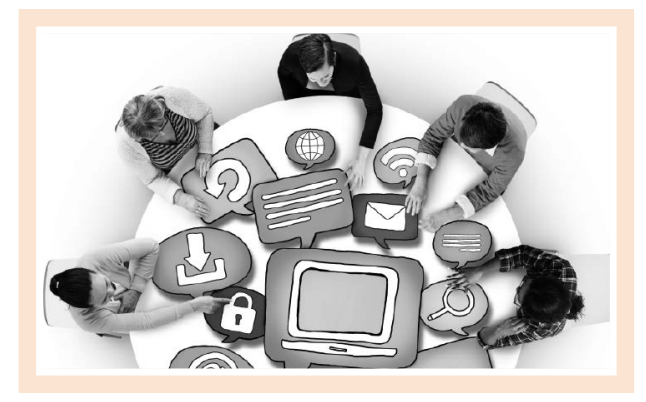

\subsubsection{Grupos de discusión}

El objetivo de esta fase consistió en asegurar que los ítems seleccionados fueran todos relevantes para los estudiantes y que no se hubieran dejado fuera otros ítems que, no siendo relevantes para las fuentes anteriores, sí lo fueran para los estudiantes. En esta fase también se comprobó la claridad de los ítems formulados para toda la muestra de estudiantes.

Se realizaron seis grupos de discusión con alumnos pertenecientes a todos los grupos de interés para el estudio. Estos alumnos ya estaban finalizando su máster, por lo que tenían criterio suficiente para definir ítems relevantes de un máster. En la organización de los mismos se tuvo en cuenta que hubiese algún representante para cada una de las variables demográficas que se querían estudiar: tipo de máster, sexo, tramos de edad, titulación de procedencia, nacionalidad, experiencia laboral y situación profesional.

En el desarrollo de las sesiones de discusión, los grupos, en una primera fase, debatieron la siguiente pregunta: «Qué factores consideráis más importantes para poder evaluar el máster como un programa de calidad». Esta discusión la realizaron sin recibir ninguna indicación externa sobre posibles indicadores.

En una segunda fase, se les facilitó a los estudiantes el cuestionario obtenido tras la revisión de los jefes de estudio (el cuestionario constaba ahora de 6 dimensiones principales, con 7 subfactores por dimensión). Los grupos de discusión debían opinar sobre este cuestionario, pudiendo eliminar aquellos ítems que no considerasen relevantes y añadir los que ellos consideraran muy importantes y no estuviesen contemplados en el listado.
Como resultado de este proceso se terminó definiendo una estructura jerárquica de dos niveles, con seis dimensiones o factores principales y seis subfactores clave en cada dimensión ( $6 \times 6)$, tamaño máximo para aplicar con garantías la metodología AHP. Estos 36 ítems son los que finalmente se consideraron relevantes para que fueran evaluados por todos los estudiantes de los másteres y cuya satisfacción se podría considerar como el paradigma de la "calidad de un máster presencial». La estructura definitiva de factores y subfactores seleccionados por estos grupos de expertos fue:

\section{Plan de estudios:}

1.1. Diseño, planificación y coordinación del programa de estudios a cursar.

1.2. Materiales didácticos.

1.3. Guías didácticas claras de las asignaturas.

1.4. Sistema de evaluación proporcionado.

1.5. Actos académicos complementarios (conferencias, visitas, etc.).

1.6. Campus virtual.

\section{Profesorado:}

2.1. Títulos académicos del profesor.

2.2. Experiencia profesional del profesor.

2.3. Habilidades de comunicación y metodología práctica.

2.4. Comportamiento justo en las evaluaciones.

2.5. Amabilidad/accesibilidad.

2.6. Conocimiento y utilización del campus.

\section{Administración y servicios de apoyo (secretaría} de estudios, etc.):

3.1. Rapidez de servicio.

3.2. Secretaría virtual (para información y peticiones).

3.3. Amabilidad/accesibilidad.

3.4. Mensajería por telefonía móvil e internet.

3.5. Amplitud de horario y facilidad para contactar

3.6. Carnet de estudiante.

\section{Bolsa de trabajo:}

4.1. Existencia de prácticas en empresas.

4.2. Existencia de ofertas profesionales (no becas). 
4.3. Amabilidad/accesibilidad.

4.4. Amplitud de horario y facilidad para contactar.

4.5. Orientación personalizada en la búsqueda de empleo.

4.6. Orientación personalizada para la creación de empresas y autoempleo.

5. Infraestructuras y equipamiento:

5.1. Comodidad de aulas y equipamiento.

5.2. Sala de informática a disposición de los alumnos.

5.3. Red wifi gratuita y acceso a internet.

5.4. Servicios de vending (bebidas, sándwiches, etc.).

5.5. Salas de trabajo en grupo.

5.6. Descuentos en aparcamientos próximos.

6. Impacto profesional futuro:

6.1. Facilidad para desarrollar nuevos programas de posgrado y doctorado.

6.2. Disponibilidad de programas de intercambio con otras universidades y escuelas de negocios extranjeras.

6.3. Acceso a la bolsa de trabajo como antiguo alumno.

6.4. Existencia de ofertas profesionales en otros países.

6.5. Descuentos como antiguo alumno en nuevos cursos y publicaciones.

6.6. Actos académicos y sociales como antiguo alumno.

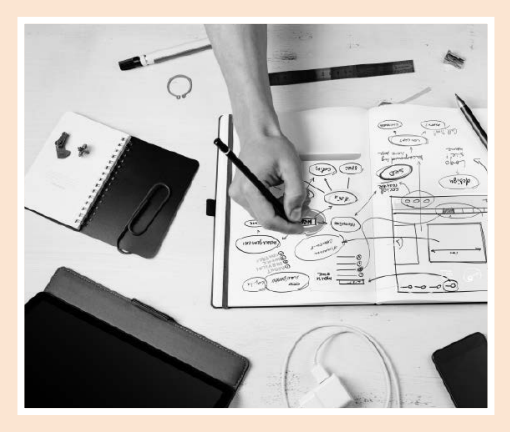

\section{La metodología AHP es un método de decisión multicriterio discreto (DMD), es decir, es un método de ayuda a la toma de decisiones, elegir o priorizar entre un conjunto finito de alternativas, cuando estas poseen muchos factores 0 atributos relevantes que incluso pueden ser contradictorios entre sí}

\subsection{Diseño, validación y aplicación del cuestio- nario}

Viswanadhan (2005) había detectado que en el análisis AHP tenía gran importancia elaborar un cuestionario para la recogida de información que redujera los esfuerzos necesarios para su cumplimentación, minimizando así la pérdida de fiabilidad en la medición. A partir de sus conclusiones se construyó el cuestionario utilizado.

Para su aplicación se redactaron unas explicaciones muy detalladas que fueron leídas por el técnico que aplicaba el cuestionario, garantizándose, así, la homogeneidad de todas las aplicaciones. Todos los cuestionarios contaban con dos ejemplos previos para el entrenamiento de los encuestados antes de realizar la valoración de los pares.

El cuestionario era anónimo y recogía las variables demográficas necesarias para el estudio: edad, sexo, nacionalidad, experiencia profesional del alumno, situación profesional actual y máster en que estaba matriculado.

Una vez elaborado el cuestionario, se realizó una validación del mismo con una aplicación-test en la que se trataba de precisar: tiempo de aplicación del cuestionario, claridad del cuestionario y existencia de posibles variables no identificadas que contaminaran su cumplimentación. Para ello, el cuestionario se aplicó a una muestra cualificada de 10 estudiantes que recogía todo el rango de características de la muestra que se quería estudiar.

Una vez elaborado y testado, el cuestionario se aplicó en el primer cuatrimestre del curso 2013/2014. 


\section{RESULTADOS}

Una vez contestados los cuestionarios y cargados los datos en Microsoft Excel 2013, se procedió, tras haber realizado el análisis de consistencia ${ }^{8}$, al cálculo de las prioridades locales y totales de los factores para cada alumno.

El interés del estudio radica en la posibilidad que aporta la metodología AHP de comparar entre sí, además de los seis factores estudiados, los factores de segundo nivel pertenecientes a cada uno de ellos. Es decir, la metodología permite establecer una única lista ordenada de los 36 subfactores (6 por factor principal), obteniéndose así el mapa de calidad esperada por los estudiantes.

\subsection{Prioridades que tienen los estudiantes respecto a los factores principales}

En la figura 5, se puede observar cómo los tres gruposobjetivo de estudiantes (españoles, dominicanos y latinoamericanos) tienen diferencias significativas en la importancia que dan a cada una de las dimensiones principales. Los estudiantes españoles se incorporan al máster con una altísima expectativa de utilidad futura, muy por encima de los estudiantes extranjeros. En esta variable, los estudiantes dominicanos (médicos estudiando gestión) traen al inicio del curso una expectativa menor.

El siguiente factor en importancia, para todos los colectivos igual, es que esperan encontrar un profesorado de gran calidad. Este aspecto se tratará más detalladamente en el punto siguiente.

\author{
(...) todos los estudiantes \\ esperan encontrar en el máster \\ profesores con experiencia, con \\ habilidades de comunicación, y \\ accesibles y cercanos. Desean \\ tener profesores que desarrollen \\ más el papel de coaches que el \\ de profesores tradicionales
}

La importancia de los dos siguientes factores aparece intercambiada entre españoles y extranjeros. Los españoles se incorporan al máster dando más importancia a la Bolsa de Trabajo que al diseño académico del programa que van a realizar, mientras que los extranjeros (dominicanos y latinoamericanos) tienen una expectativa sobre la Bolsa de Trabajo algo menor, dando más importancia al diseño académico que van a acometer, probablemente por las dificultades que en los últimos años tienen estos estudiantes para acceder al mercado laboral.

El valor de las infraestructuras y equipamientos y los servicios y apoyos administrativos no aporta valor añadido a ninguno de los tres grupos de estudiantes analizados al inicio del máster.

\footnotetext{
${ }^{8}$ Este trabajo está fundamentado en la investigación realizada por el autor, bajo la dirección de los doctores don Miguel Martín Dávila y doña Diana Pérez-Bustamante Yábar, y que culminó con la realización de una tesis doctoral (Pastor, 2015). En dicha tesis se encuentra detallado todo el proceso matemático seguido en la aplicación de la metodología AHP, incluyendo la justificación y el proceso de realización del cálculo de las prioridades locales, siguiendo el método del autovector principal (Moreno, 2002; Saaty, 1980 y 1998), el cálculo de las matrices normalizadas utilizando el método distributivo (Saaty, 1980 y 1998 ; Zanazzi, 2003), las prioridades globales obtenidas por composición jerárquica (Saaty, 1980 y 1998; Zanazzi, 2003) y las prioridades totales o mapa de calidad esperada, calculadas mediante la forma lineal de suma ponderada (Saaty, 1980 y 1998; Zanazzi, 2003), si bien en nuestro caso, al no compartir factores de segundo orden ninguno de los criterios principales, las prioridades globales y totales coincidirán. Por último, se realizó la agregación de las prioridades locales y globales obtenidas para cada uno de los decisores a través del cálculo de la media geométrica de las mismas (Moreno, 2002; Saaty, 1980 y 1998; Zanazzi, 2003).

Previamente a estos cálculos, se había realizado un detallado análisis de consistencia aplicado a las decisiones individuales y un estudio comparado para observar el impacto que, sobre las prioridades totales de los estudiantes, tenía el número de cuestionarios aceptados según se utilizaran diversos valores de razón de consistencia ( $R C \geq 0,1,0,15,0,2$ y 0,25). Pudo observarse que no se alteró en ninguno de los casos el orden de prioridad de los factores (siendo muy leve, cualitativamente hablando, la intensidad de las variaciones en los cinco factores principales. Por este motivo, para el cálculo de las prioridades globales se tuvieron en cuenta todos los cuestionarios con objeto de no perder riqueza en el número de opiniones, siguiendo el criterio de algunos autores de que el ser humano es inconsistente por naturaleza, especialmente cuando tiene que elegir entre un amplio abanico de alternativas, como era nuestro caso, y, por tanto, deberian ser tenidos en cuenta tanto los juicios consistentes que formulara el decisor, como los inconsistentes (Zanazzi, 2003).
}

En este trabajo, dada la complejidad y necesaria extensión que hubiera requerido detallar estos aspectos, se han obviado los mismos en aras de la claridad y sencillez del objetivo del mismo. 
Figura 5. Prioridades para los factores principales: toda la muestra y desglosadas por grupos-objetivo

\section{Ejemplo de prioridades ohtenidlas en los Factores Prineipales}

\section{Todala muestra}
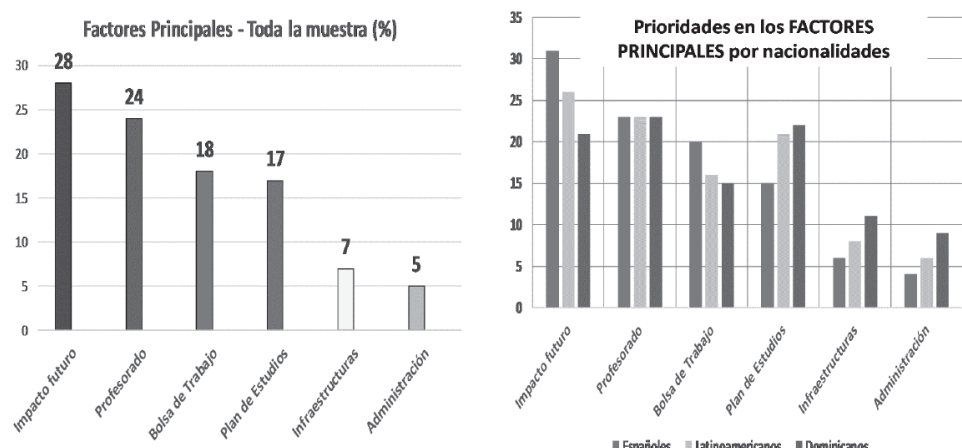

|| Españoles || Latinoamericanos || Deminiconos

Fuente:

\subsection{Un profesorado de calidad}

La figura 6 recoge cómo todos los estudiantes están de acuerdo en resaltar la gran importancia que tiene para ellos la capacidad pedagógica del profesor, su experiencia profesional (sobre todo para los estudiantes españoles) y una actitud justa (más importante para los estudiantes latinoamericanos). También conviene hacer notar que para todos los estudiantes de másteres, 10 que menos les interesa de un profesor son sus títulos académicos.

Estos datos parecen indicar una diferencia notable de criterios entre lo que la Administración universitaria valora para considerar a un profesor como un docente de calidad $y$ lo que piensan los estudiantes en los programas máster.

\subsection{Prioridades que tienen los estudiantes respecto a los 36 factores en su conjunto (mapa de calidad esperada)}

En el anexo se detallan las prioridades que para cada uno de estos colectivos tienen los 36 factores analizados.

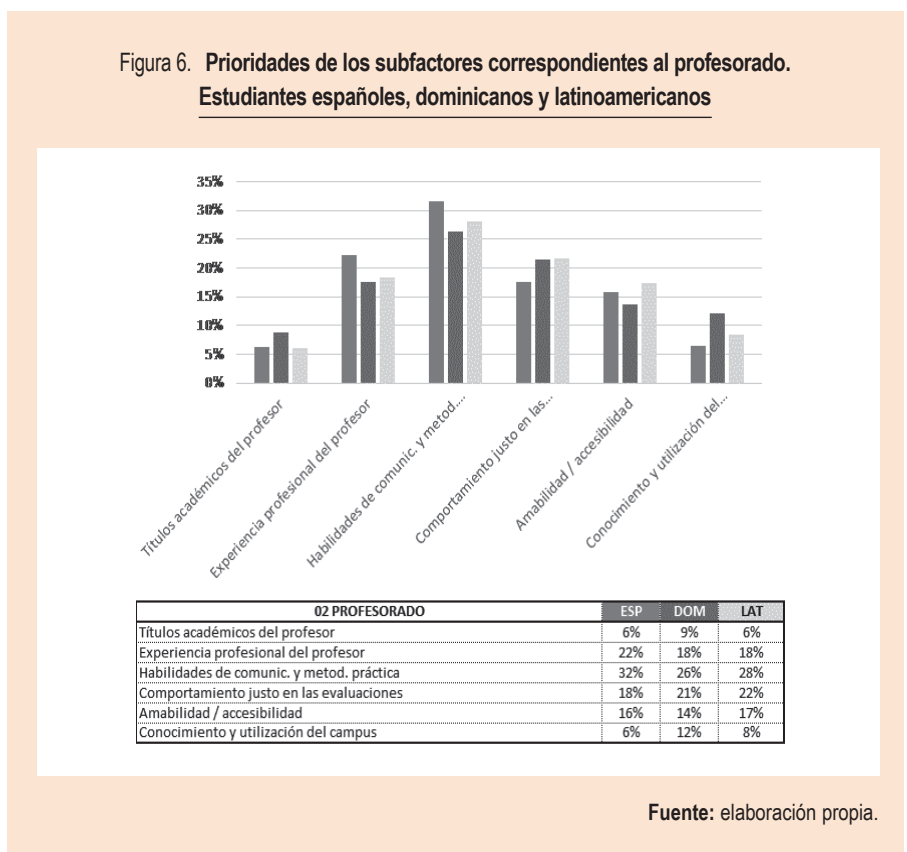




\subsection{Comparación de las 10 prioridades principales de los tres grupos de es- tudiantes}

En la tabla 2 puede observarse, comparadas, las 10 prioridades principales de cada grupo-objetivo de estudiantes. La tabla hace ver todo lo que tienen en común estos tres grupos, ya que, en solo 14 ítems, están recogidas las 10 prioridades principales de todos ellos. Y la tabla también recoge cómo estas 10 prioridades principales explican prácticamente el $50 \%$ de la calidad que esperan recibir los estudiantes (en el caso de los estudiantes españoles, casi el $60 \%$ de la calidad esperada está recogida en 10 factores).

Para los españoles, las oportunidades profesionales que pueda ofrecer el máster superan a las expectativas que tienen sobre el profesorado, pero, en general, para todos los alumnos, esta variable es prácticamente la más importante. Y en el estudio, son los propios estudiantes los que nos dicen qué es para ellos un profesorado de calidad. Escucharlos o no ya es cuestión de cada institución educativa.

Los estudiantes latinoamericanos comparten con los españoles estas expectativas sobre el profesorado y, además, tienen mayor interés sobre el plan de estudios que van a acometer que sus compañeros españoles, para los que no figura ningún factor del diseño del programa entre sus 10 prioridades principales.

En la figura 7 se pueden observar los seis factores que todos los estudiantes, sean de donde sean, están de acuerdo en incluir entre sus 10 principales prioridades. Tres son relativas a la calidad del profesorado. Este cuadro sería la esencia de la calidad que esperan recibir.

\section{Tabla 2. Prioridades totales principales en los tres grupos de estudiantes}

\begin{tabular}{|c|c|c|c|}
\hline \multirow[b]{4}{*}{ Prioridades } & ESPAÑOLES & LATINOAMERICANOS & DOMINICANOS \\
\hline & \multicolumn{3}{|c|}{$\begin{array}{c}\text { Estas } 10 \text { prioridades suponen para este colectivo el siguiente } \\
\text { porcentaje de todas sus prioridades }\end{array}$} \\
\hline & $57 \%$ & $52 \%$ & $45 \%$ \\
\hline & & Orden de prioridad & \\
\hline \multicolumn{4}{|c|}{ INTERÉS EN LA PROYECCIÓN PROFESIONAL FUTURA } \\
\hline Acceso a la Bolsa de Trabajo como Antiguo Alumno. & 1 & 3 & 10 \\
\hline Posibilidad de ofertas trabajo extranjero. & 2 & 1 & 3 \\
\hline Existencia de Ofertas Profesionales (no becas). & 4 & 9 & \\
\hline Intercambios en el extranjero. & 7 & 6 & 7 \\
\hline Orientación personal en búsqueda de empleo & 8 & & \\
\hline Existencia de Prácticas en empresas. & 9 & & \\
\hline Nuevos programas de postgrado y doctorado. & & & 5 \\
\hline \multicolumn{4}{|c|}{ INTERÉS EN LA CALIDAD DEL PROFESOR } \\
\hline Habilidades de comunicación y metodología práctica del profesor. & 3 & 2 & 1 \\
\hline Experiencia profesional del profesor. & 5 & 7 & 8 \\
\hline Comportamiento justo en las evaluaciones. & 6 & 5 & 4 \\
\hline Accesibilidad del profesor. & 10 & 8 & \\
\hline \multicolumn{4}{|c|}{ INTERÉS EN EL DISEÑO ACADÉMICO } \\
\hline Diseño, planificación y coordinación del programa académico. & & 4 & 2 \\
\hline Guías didácticas claras de las asignaturas. & & 10 & 6 \\
\hline Materiales didácticos. & & & 9 \\
\hline
\end{tabular}


Figura 7. Prioridades que todos los estudiantes comparten dentro de sus 10 principales preferencias. El núcleo de las expectativas de calidad en esta escuela de negocios y universidad

\section{Ėemplo de antálisis: lo uue más valoran en comín todios los grupos}

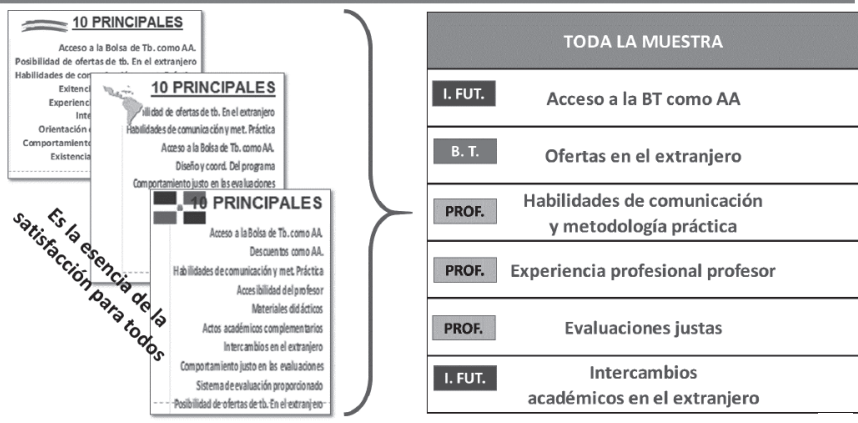

\section{CONCLUSIONES}

\subsection{Respecto a la idoneidad del empleo de las metodologías despliegue de la función de calidad y proceso analítico jerárquico para el diseño de programas formativos}

El estudio realizado ha permitido comprobar la utilidad de emplear las metodologías QFD y AHP para el diseño de programas formativos, en este caso, de educación superior, si queremos incorporar al mismo la opinión de diversos agentes cualificados: profesores, empresarios, estudiantes, Gobiernos, agentes sociales, etc.

\subsection{Respecto a las preferencias manifestadas por los estudiantes de lo que esperan en- contrar en un máster}

El estudio refleja cómo prácticamente todos los estudiantes de los másteres presenciales de esta escuela de negocios y universidad tienen una prioridad esencial casi idéntica: el objetivo de realizar un máster es mejorar la posición del alumno en el mercado laboral. Entrar en el mercado laboral, si estoy fuera, y mejorar o impedir que me expulsen, si ya estoy dentro. Los estudiantes perciben los másteres como herramientas de empleabilidad, más que como herramientas de contenidos académicos.
Pero los estudiantes saben que son y quieren ser trabajadores del conocimiento. ¿Qué aspectos diferencian ese contenido de ser conocimiento? Los estudiantes lo tienen claro, la capacidad para aplicar exitosamente los contenidos estudiados: la experiencia. Por eso, todos los estudiantes esperan encontrar en el máster profesores con experiencia, con habilidades de comunicación, y accesibles y cercanos. Desean tener profesores que desarrollen más el papel de coaches que el de profesores tradicionales.

Estas dos conclusiones eran, en gran medida, conocidas por todos (excepto por la autoridad universitaria que sigue considerando que la principal virtud de un profesor de máster es que sea doctor). La aportación del trabajo en esta línea es cuantificar la importancia de este sentimiento. El que el estudio sea un caso práctico concreto, en una escuela de negocios y universidad concreta y en un curso académico determinado, desde luego no permite la generalización a toda la población de estudiantes de másteres, pero no por eso quita relevancia a los datos obtenidos. Es más, los datos aportados son solo un indicador, pero son por desgracia un indicador bastante dramático del alejamiento de las autoridades académicas españolas de las necesidades sociales. 


\subsection{Conclusiones y líneas futuras de investigación sobre estrategias competitivas para escuelas de negocios y universidades privadas}

La incorporación masiva de los jóvenes a la universidad ${ }^{9}$ está generando que los estudios de grado en el siglo XXI se estén convirtiendo en formación básica, semejante a lo que fue el bachillerato en España en la segunda mitad del siglo XX.

Si a esta masificación, se une la bajísima preparación general que trae el alumno procedente del sistema educativo español de secundaria (véanse datos del Informe PISA, Ministerio de Educación Cultura y Deporte, 2014b) y la particular aplicación que se ha hecho en España del Proceso de Bolonia, el resultado ha sido que la formación de graduados no esté siendo todo lo exigente que la sociedad podría necesitar (Hernández, Delgado y Pericay, 2013; Núñez, 2013).

Para compensar esta carencia de nivel profesional en la formación de grado, los estudiantes precisarán continuar sus estudios con algún programa de posgrado, normalmente un máster. Es decir, el EEES lo que está haciendo es transformar la enseñanza universitaria en una formación de posgrado (De Carreras, 2013). Así, los másteres aumentarán progresivamente su importancia para la empleabilidad de los egresados y se convertirán en la formación que dé realmente visibilidad al currículo del estudiante, por lo que, como se refleja en los resultados del estudio, los estudiantes elegirán el centro educativo por la eficacia que demuestre en la adquisición de competencias profesionales y la empleabilidad en sus estudiantes ${ }^{10}$.

En este escenario tan altamente competitivo, la universidad pública tendrá serias dificultades para responder con flexibilidad y rapidez a la hora de satisfacer adecuadamente lo que los estudiantes necesitan y exigen. La burocracia, la falta de flexibilidad para poder seleccionar los profesores más adecuados para sus programas (doctores vs. profesionales), un fuerte poder sin- dical que no permite incorporar la valoración individual del desempeño y un inevitable clientelismo político con las Administraciones autonómicas de las que dependen financieramente, dificulta, y casi impide, la renovación de las estructuras que se requerirían para poder impartir programas másteres de reconocido prestigio y con capacidad para atraer alumnos de todo el mundo (Hernández, Delgado y Pericay, 2013; Núñez, 2013).

Sin embargo, las escuelas de negocios, con una larga trayectoria de prestigio y reconocimiento social (De Carrera, 2013; Núñez, 2013), estrechamente unidas a universidades privadas, sin estar sometidas a las servidumbres de la universidad pública (más allá de las exigencias derivadas de las acreditaciones de sus programas oficiales) y con una marcada orientación a considerar al alumno y al tejido empresarial como cliente principal de su esfuerzo, se encuentran en una excelente situación de ventaja competitiva para liderar y desarrollar unos posgrados que aseguren su calidad (satisfacción de sus necesidades) a los estudiantes.

Los estudiantes esperan tener buenos profesores y que el máster les sirva para su mejora profesional. Y existen importantísimas diferencias entre las cualidades que los estudiantes atribuyen a un buen profesor $y$ los méritos que consideran las autoridades universitarias para los mismos.

Probablemente sea esa flexibilidad para cambiar el enfoque de la «gestión por ratios» a la «creación de valor para el estudiante» la que permitirá a las escuelas de negocios y universidades privadas mantener su capacidad de innovación en este sector.

No deberíamos perder de vista el «principio de la reina roja» ${ }^{11}$ si queremos tener una universidad de calidad.

\footnotetext{
${ }^{9}$ En el curso 2013/2014, la tasa neta de escolarización en educación universitaria fue del 28,6\% según datos del Ministerio de Educación, Cultura y Deporte (2014a). Según el informe Eurydice brief. Modernisation of higher education in Europe: access, retention and employability (2014), uno de los cinco objetivos estratégicos de la Comisión Europea para la educación superior es que, para el año 2020, el $40 \%$ de la población de 30 a 34 años deberá tener una educación superior 0 equivalente.

${ }^{10}$ En España hay 83 universidades (50 públicas y 33 privadas; de todas ellas, 6 son no presenciales). Según datos del Ministerio de Educación, Cultura y Deporte (2014a), cada año se crea una nueva universidad privada en España.

${ }^{11}$ Este principio lo formuló en 1973 el biólogo Leigh Van Valen para explicar cómo las especies necesitaban estar en un proceso de mejora permanente para mantener la posición que ya habian adquirido. Van Valen toma el concepto del libro que Lewis Carroll publicó en 1871, Alicia a través del espejo y lo que Alicia encontró al otro lado. En el cuento, tras una alocada carrera de Alicia junto a la reina Roja, Alicia observa sorprendida que están en el mismo sitio, a lo que la reina roja le contesta: «Lo que es aquí, como ves, hace falta correr todo cuanto una pueda para permanecer en el mismo sitio. Si se quiere llegar a otra parte hay que correr por lo menos dos veces más rápido» (Carroll, 1979, pág. 62).
} 


\section{LÍNEAS FUTURAS DE INVESTIGACIÓN}

De estas conclusiones se sugieren varias líneas futuras de investigación:

- Estudiar cómo se modifican las expectativas de los estudiantes al terminar sus estudios de máster para detectar si, a lo largo del proceso formativo, sus expectativas sufren cambios relevantes. Este conocimiento permitiría ajustar tanto el diseño académico como la definición y racionalización de las expectativas de los estudiantes al comienzo del máster. Ya hemos visto en el punto anterior la utilidad que tienen las metodologías empleadas en el estudio para este aspecto.

- Replicar este estudio con los estudiantes de programas máster on-line. La formación no presencial es ya el presente (véase figura 8) y el futuro de la formación universitaria en gran medida. Conocer cuáles son las expectativas y prioridades de un estudiante a distancia y qué diferencias transculturales existen permitiría diseñar programas de mayor calidad.

- Estas líneas deberían incluir específicamente grupos-objetivo de estudiantes latinoamericanos y, muy en especial, dominicanos.
- Estas líneas podrían complementarse con un estudio longitudinal al cabo de $3 \circ 5$ años, para determinar con egresados tanto la utilidad del máster como de los indicadores definidos.

- Ampliar la investigación completando la identificación y selección de los factores clave que deberán ser posteriormente evaluados. Este conocimiento permitiría afinar en el diseño del mapa de calidad esperado.

- Ampliar la investigación a los empleadores y profesionales del tejido empresarial para aumentar la potencia del máster como herramienta de posicionamiento laboral.

- Ampliar esta investigación considerando al Gobierno de la República Dominicana, en concreto a las autoridades sanitarias y al Ministerio para la Educación Superior, la Ciencia y la Tecnología (MESCYT), como «actores prescriptores del proceso».

Figura 8. Tasa de variación de los estudiantes en grado entre 2003-2004 y 2013-2014

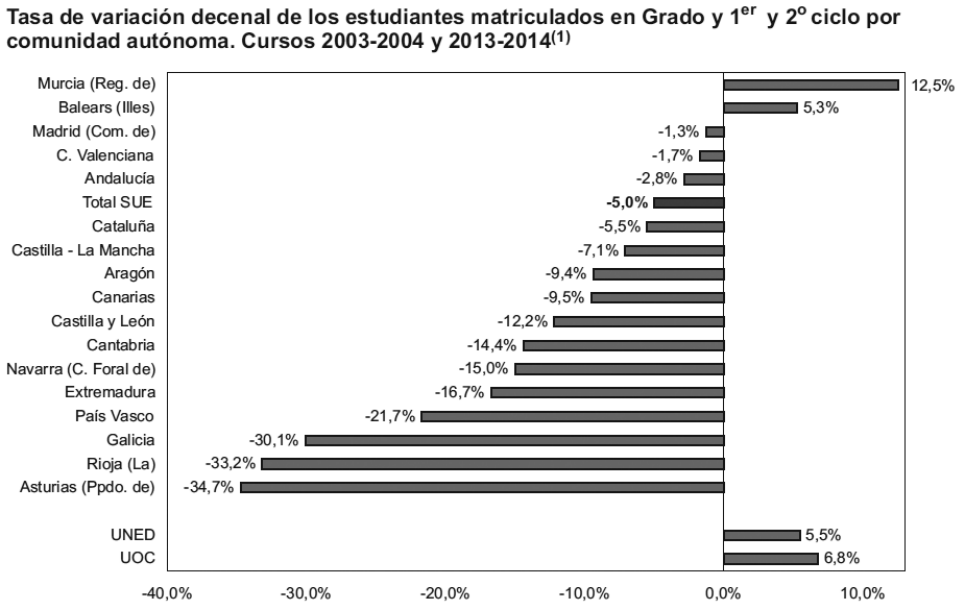

Fuente: Ministerio de Educación, Cultura y Deporte (2014a). 


\section{BIBLIOGRAFÍA}

Agencia de Calidad, Acreditación y Prospectiva (ACAP) de las Universidades de Madrid [2006]: Estudio internacional sobre criterios e indicadores de calidad en las universidades, Madrid: ACAP.

Asociación Latinoamericana de QFD (QFDLAT) [2014]: AHP (analytic hierarchy process). Disponible en: http://www.qfdlat.com/Herramientas_QFD/herramientas_qfd.html\#AHP.

Carreras, F. de [2013]: «Hacia otra universidad», en J. Hernández, A. Delgado y X. Pericay (eds.), La universidad cercada: testimonios de un naufragio, España: Anagrama, págs. 81-106.

Carroll, L. [1979]: Alicia a través del espejo y lo que Alicia encontró al otro lado, Madrid: Alianza Editorial.

European Commission (Education, Audiovisual and Culture Executive Agency) [2015]: Modernisation of higher education in Europe: access, retention and employability (2014). (EURYDICE BRIEF No. 180/EN). DOI: $10.2797 / 60697$.

European Education Information Network (EURYDICE) [2014]: Tackling early leaving from education and training in Europe: strategies, policies and measures.

Gasalla, J. M. ${ }^{a}$ [2010]: Marketing de la formación de directivos, Pozuelo de Alarcón: Pirámide.

Guzmán, A. [2013]: Introducción al QFD (quality function deployment) despliegue de la función de calidad. Disponible en: http://www.itsao.edu.mx/default/Revista/ Publicaciones/ARTICULO\%20(QFD).pdf.

Hernández, J.; Delgado, A. y Pericay, X. (eds.) [2013]: La universidad cercada: testimonios de un naufragio, España: Anagrama.

Jimeno, J. [2013]: Despliegue de la función de calidad (QFD): guía de uso. Para qué sirve el QFD y cómo realizarlo. Disponible en: http://www.pdcahome. com/1932/qfd-despliegue-calidad/

Johnson, C. N. [2003]: «QFD explicado», Quality Progress, 2001.

Kuo, W. [2006]: «Assessment for US engineering programs», IEEE Transactions on Reliability, 55 (1), pág. 1-6. Doi:10.1109/TR.2005.863791.
Marsh, H. W. y Roche, L. A. [1997]: «Making students' evaluations of teaching effectiveness effective», American Psychologist, 52 (11), págs. 1.187-1.197.

Ministerio de Educación Cultura y Deporte [2014a]: Datos y cifras del sistema universitario español 2014/2015, Secretaría General Técnica.

[2014 b]: Datos del Informe PISA-Resolución de problemas. Disponible en: http://www.mecd.gob.es/prensa-mecd/actualidad/2014/04/20140401-pisa.html.

Moreno, J. [2002]: «El proceso analítico jerárquico. Fundamentos, metodología y aplicaciones», RECTA Monográfico, 1, págs. 21-53.

Moreno, J.; Aguaron, J.; Cano, F. y Escobar, M. ${ }^{a}$ T. [1998]: «Validez, robustez y estabilidad en decisión multicriterio. Análisis de sensibilidad en el proceso analítico jerárquico», Revista de la Real Academia de Ciencias Exactas, Físicas y Naturales, 92 (4), pág. 387-397.

Núñez, C. E. [2013]: Universidad y ciencia en España (claves de un fracaso y vías de solución), Madrid (España): Gadir.

Owlia, M. S. y Aspinwall, E. M. [1998]: «Application of quality function deployment for the improvement of quality in an engineering department», European Journal of Engineering Education, 23 (1), págs. 105-115.

Pastor, A. [2015]: Aplicación de las técnicas despliegue de la función de calidad (QFD) y proceso analítico jerárquico $(A H P)$ en el diseño de la oferta de estudios de postgrado: un análisis transcultural, tesis doctoral no publicada, Universidad Rey Juan Carlos, Madrid.

QFD Institute [2013]: The official source for QFD. Disponible en: http://www.qfdi.org/.

Raharjo, H.; Xie, M.; Ngee, T. y Brombacher, A. [2007]: «A methodology to improve higher education quality using the quality function deployment and analytic hierarchy process», Quality Management, 18 (10), págs. 1.097-1.115.

Sa, P. y Saraiva, P. [2001]: «The development of an ideal kindergarten through concept engineering/quality function deployment», Total Quality Management, 12 (3), págs. 365-372. 
Saaty, T. L. [1980]: The analytic hierarchy process: planning, priority setting, resources allocation, New York: McGraw-Hill.

[1998]: «Método analítico jerárquico (AHP): principios básicos», en E. Martínez y M. Escudey (eds.), Evaluación y decisión multicriterio: reflexiones y experiencias, Santiago de Chile: Editorial Universidad.

Saaty, T. L. y Vargas, L. G. [2001]: «The seven pillars of the analytic hierarchy process», en T. L. Saaty y L. G. Vargas, Models, methods, concepts \& applications of the analytic hierarchy process, Springer, págs. 27-46.

Singh, V.; Grover, S. y Kumar, A. [2008]: «Evaluation of quality in an educational institute: a quality function deployment approach», Educational Research and Review, 3(4), págs. 162-168.

Tsinidou, M.; Gerogiannis, V. y Fitsilis, P. [2010]: «Evaluation of the factors that determine quality in higher education: an empirical study», Quality Assurance in Education, 18(3), págs. 227-244.

Valor, J. y Ribera, J. [1988]: «Algunos aspectos de gestión en instituciones sanitarias», CIRIEC-España: Revista de Economía Pública, Social y Cooperativa, 4, págs. 175-203.

Viswanadhan, K. [2005]: «How to get responses for multi-criteria decisions in engineering education-an AHP based approach for selection of measuring instrument», Financial Support, págs. 20-28.

Zanazzi, J. L. [2003]: «Anomalías y supervivencia en el método de toma de decisiones de Saaty», Problemas del Conocimiento en Ingeniería y Geología, 1, págs. 148-170.

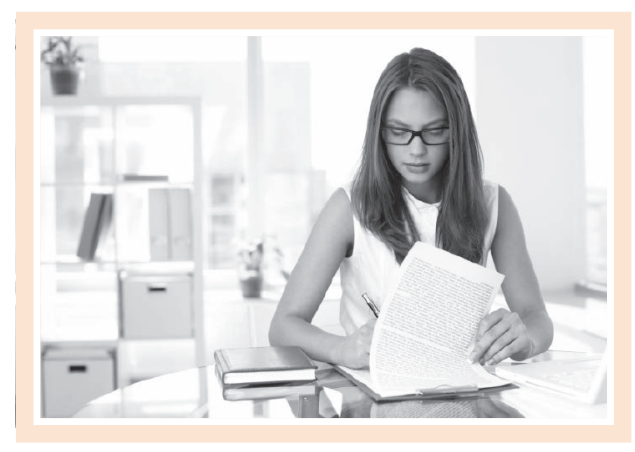

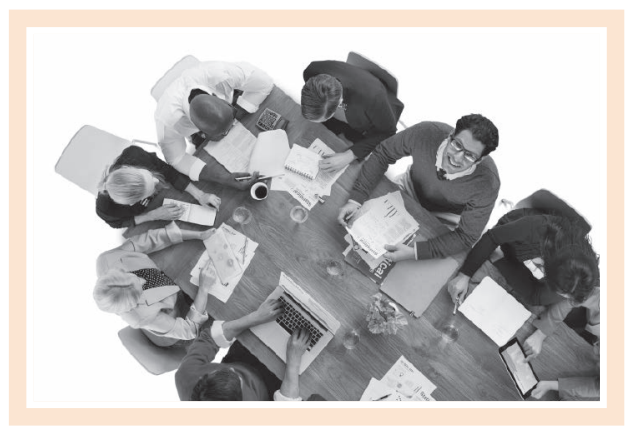

\section{ANEXOS}

\section{Prioridades totales de los estu- diantes españoles, latinoameri- canos y dominicanos}

\begin{tabular}{|l|c|}
\hline PRIORIDADES (\%) TOTALES DE LOS ESTUDIANTES ESPAÑOLS \\
\hline Acceso a la Bolsa de Trabajo como Ant. Al. & 8,5 \\
\hline Posibilidad de ofertas trabajo extranjero & 8,1 \\
\hline Habilidades de comunic. y metod. práctica & 7,6 \\
\hline Exitencia de Ofertas Profesionales (no becas) & 6,9 \\
\hline Experiencia profesional del profesor & 5,4 \\
\hline Intercambios en el extranjero & 4,5 \\
\hline Orientación personal en búsqueda de empleo & 4,4 \\
\hline Comportamiento justo en las evaluaciones & 4,3 \\
\hline Existencia de Prácticas en empresas & 4,1 \\
\hline Accesibilidad del profesor & 3,8 \\
\hline Diseño, planificación y coordinación del progr. & 3,7 \\
\hline Descuentos como Ant. Al. & 3,6 \\
\hline Materiales didácticos & 3,5 \\
\hline Nuevos programas de postgrado y doctorado & 3,2 \\
\hline Orientación personal para crear empresas & 2,9 \\
\hline Sistema de evaluación proporcionado & 2,4 \\
\hline Actos académicos y sociales como Ant. Al. & 2,2 \\
\hline Guías didácticas claras de las asignaturas & 2,1 \\
\hline Actos académicos complementarios & 1,7 \\
\hline Conocimiento y utilización del campus & 0,6 \\
\hline Comodidad de aulas y equipamiento & 0,4 \\
\hline Títulos académicos del profesor & 1,6 \\
\hline Campus virtual & 1,5 \\
\hline Amplitud de horario y facilidad para contactar & 1,5 \\
\hline Salas de Trabajo en grupo & 1,4 \\
\hline Accesibilidad Bolsa de Trabajo & 1,3 \\
\hline Sala de informática de uso libre & 1,3 \\
\hline Red wifi gratuita y acceso a internet & 1,2 \\
\hline Amplitud de horario y facilidad para contactar & 1,2 \\
\hline Rapidez de Servicio (secretaría) & 1,1 \\
\hline Accesibilidad Secretaría & 1,1 \\
\hline Secretaría virtual (información y gestiones) & 0,9 \\
\hline Mensajería por telefonía móvil e internet & 0,8 \\
\hline Servicios de vending (bebidas, sandwich...) & 0,7 \\
\hline Descuentos en aparcamientos próximos & 0,6 \\
\hline Carnet de Estudiante & 1,7 \\
\hline TOTAL & \\
\hline
\end{tabular}


Aplicación de las técnicas despliegue de la función de calidad (QFD) y proceso analítico jerárquico (AHP) ～～～A. Pastor Sanmillán a la mejora de la calidad de la formación de posgrado

\begin{tabular}{|l|c|}
\hline PRIORIDADES (\%) TOTALES DE LOS ESTUDIANTES DOMINICANOS \\
\hline Habilidades de comunic. y metod. práctica & 5,8 \\
\hline Diseño, planificación y coordinación del progr. & 5,2 \\
\hline Posibilidad de ofertas trabajo extranjero & 4,8 \\
\hline Comportamiento justo en las evaluaciones & 4,7 \\
\hline Nuevos programas de postgrado y doctorado & 4,4 \\
\hline Guías didácticas claras de las asignaturas & 4,3 \\
\hline Intercambios en el extranjero & 4,1 \\
\hline Experiencia profesional del profesor & 3,9 \\
\hline Materiales didácticos & 3,7 \\
\hline Acceso a la Bolsa de Trabajo como Ant. Al. & 3,6 \\
\hline Orientación personal para crear empresas & 3,5 \\
\hline Sistema de evaluación proporcionado & 3,5 \\
\hline Exitencia de Ofertas Profesionales (no becas) & 3,2 \\
\hline Orientación personal en búsqueda de empleo & 3,1 \\
\hline Existencia de Prácticas en empresas & 3,1 \\
\hline Accesibilidad del profesor & 3,0 \\
\hline Conocimiento y utilización del campus & 2,7 \\
\hline Descuentos como Ant. Al. & 2,6 \\
\hline Actos académicos complementarios & 1,5 \\
\hline Campus virtual & 2,5 \\
\hline Red wifi gratuita y acceso a internet & 2,5 \\
\hline Comodidad de aulas y equipamiento & 1,1 \\
\hline Salas de Trabajo en grupo & 2,4 \\
\hline Sala de informática de uso libre & 2,4 \\
\hline Amplitud de horario y facilidad para contactar & 2,4 \\
\hline Títulos académicos del profesor & 2,4 \\
\hline Rapidez de Servicio (secretaría) & 2,0 \\
\hline Accesibilidad Secretaría & 1,9 \\
\hline Secretaría virtual (información y gestiones) & 1,7 \\
\hline Actos académicos y sociales como Ant. Al. & 1,7 \\
\hline Mensajería por telefonía móvil e internet & 1,5 \\
\hline Amplitud de horario y facilidad para contactar & 1,4 \\
\hline Accesibilidad Bolsa de Trabajo & 1,3 \\
\hline Carnet de Estudiante & 1,3 \\
\hline Servicios de vending (bebidas, sandwich...) & 1,7 \\
\hline Descuentos en aparcamientos próximos & \\
\hline TOTAL & \\
\hline & \\
\hline
\end{tabular}

\begin{tabular}{|c|c|}
\hline \multicolumn{2}{|c|}{ PRIORIDADES (\%) TOTALES DE LOS ESTUDIANTES LATINOAMERICANOS } \\
\hline Posibilidad de ofertas trabajo extranjero & 6,8 \\
\hline Habilidades de comunic. y metod. práctica & 6,7 \\
\hline Acceso a la Bolsa de Trabajo como Ant. Al. & 6,5 \\
\hline Diseño, planificación y coordinación del progr. & 5,5 \\
\hline Comportamiento justo en las evaluaciones & 5,2 \\
\hline Intercambios en el extranjero & 4,8 \\
\hline Experiencia profesional del profesor & 4,4 \\
\hline Accesibilidad del profesor & 4,2 \\
\hline Exitencia de Ofertas Profesionales (no becas) & 4,1 \\
\hline Guías didácticas claras de las asignaturas & 4,0 \\
\hline Descuentos como Ant. Al. & 3,7 \\
\hline Existencia de Prácticas en empresas & 3,5 \\
\hline Sistema de evaluación proporcionado & 3,2 \\
\hline Materiales didácticos & 3,1 \\
\hline Orientación personal para crear empresas & 3,0 \\
\hline Orientación personal en búsqueda de empleo & 2,9 \\
\hline Nuevos programas de postgrado y doctorado & 2,7 \\
\hline Campus virtual & 2,3 \\
\hline Actos académicos complementarios & 2,3 \\
\hline Comodidad de aulas y equipamiento & 2,0 \\
\hline Red wifi gratuita y acceso a internet & 2,0 \\
\hline Conocimiento y utilización del campus & 2,0 \\
\hline Salas de Trabajo en grupo & 1,6 \\
\hline Actos académicos y sociales como Ant. Al. & 1,6 \\
\hline Sala de informática de uso libre & 1,5 \\
\hline Títulos académicos del profesor & 1,5 \\
\hline Amplitud de horario y facilidad para contactar & 1,4 \\
\hline Rapidez de Servicio (secretaría) & 1,1 \\
\hline Amplitud de horario y facilidad para contactar & 1,1 \\
\hline Accesibilidad Bolsa de Trabajo & 1,1 \\
\hline Accesibilidad Secretaría & 1,1 \\
\hline Mensajería por telefonía móvil e internet & 0,8 \\
\hline Secretaría virtual (información y gestiones) & 0,8 \\
\hline Servicios de vending (bebidas, sandwich...) & 0,8 \\
\hline Carnet de Estudiante & 0,5 \\
\hline Descuentos en aparcamientos próximos & 0,5 \\
\hline TOTAL & 100 \\
\hline
\end{tabular}

\section{Relación de los 42 trabajos analizados por el empleo de las metodolo- gías QFD + AHP al ámbito universitario}

Debido a su extensión (4 págs.) puede consultarse en:

https://drive.google.com/file/d/0B8YafBplGeHwbkM2Z1JPdkFzVGM/view?usp=sharing 